TRANSACTIONS OF THE

AMERICAN MATHEMATICAL SOCIETY

Volume 349, Number 5, May 1997, Pages 1919-1943

S 0002-9947(97)01856-4

\title{
HIGHER-DIMENSIONAL VIRTUAL DIAGONALS AND IDEAL COHOMOLOGY FOR TRIANGULAR ALGEBRAS
}

\author{
ALAN L.T. PATERSON AND ROGER R. SMITH
}

\begin{abstract}
We investigate the cohomology of non-self-adjoint algebras using virtual diagonals and their higher-dimensional generalizations. We show that infinite dimensional nest algebras always have non-zero second cohomology by showing that they cannot possess 2-virtual diagonals. In the case of the upper triangular atomic nest algebra we exhibit concrete modules for non-vanishing cohomology.
\end{abstract}

\section{INTRODUCTION}

Hochschild cohomology for a Banach algebra $A$ was developed by B.E. Johnson [13] from the point of view of cocycles. Another approach to Banach algebra cohomology theory has been developed by A. Helemskii and his school. This treats this theory as a relative homology theory (in the sense of Eilenberg and Moore [6]). A key role in this theory is played by the functor Ext, and for a Banach $A$-bimodule $X$, the Johnson cohomology groups $H^{n}(A, X)$ are shown to be $\operatorname{Ext}_{A^{e}}\left(A_{+}, X\right)$, where $A_{+}$is $A$ with a unit adjoined and $A^{e}=A \widehat{\otimes} A^{o p}[12$, p. 155]. An invaluable account of the theory is given in the book [12] of Helemskii. The basic definitions and results that we will need are presented in $\S 2$.

In [13], Johnson introduced the important property of amenability for $A$. The Banach algebra $A$ is called amenable if $H^{1}\left(A, X^{*}\right)=0$ for every Banach $A$-bimodule $X$. A beautiful result of Helemskii and Scheinberg ([12], p. 254) asserts that $A$ is amenable if and only if $A_{+}^{*}$ is an injective $A$-bimodule. Amenability for a $C^{*}$ algebra is equivalent to nuclearity, and an appropriate version of amenability for a von Neumann algebra is equivalent to the latter's injectivity (in the usual sense of operator algebras). These results are mostly due to [11] and [2], [3]. The reader is referred to [18] for an account of cohomology in the self-adjoint setting.

Johnson showed that the amenability of a Banach algebra $A$ is equivalent to the existence of a certain element of $A^{* *}$, called a virtual diagonal. For convenience, let $A$ be unital with unit $e$. (Throughout this paper, $A$ will be assumed unital.) Then a virtual diagonal for $A$ is an element $M \in(A \widehat{\otimes} A)^{* *}$ such that $a M=M a$ for all $a \in A$ and $\pi^{* *}(M)=e$, where $\pi: A \widehat{\otimes} A \rightarrow A$ is the product map. It is often convenient to construct a virtual diagonal in proving the amenability of $A$, since

Received by the editors July 10, 1995 and, in revised form, November 15, 1995.

1991 Mathematics Subject Classification. Primary 47D25, 46H25.

Key words and phrases. Cohomology, nest algebra, operator algebra, diagonal, virtual diagonal, cocycle.

Partially supported by grants from the National Science Foundation. 
then we do not have to consider all Banach dual $A$-bimodules $X^{*}$ and show that $H^{1}\left(A, X^{*}\right)=0$.

The higher-dimensional version of amenability for a (unital) Banach algebra $A$ was investigated by Effros and Kishimoto ([5], §3). Let us call $A$ n-amenable $(n \geq 1)$ if $H^{n}\left(A, X^{*}\right)=0$ for all Banach $A$-bimodules $X$. Effros and Kishimoto showed that $n$-amenability for $A$ is equivalent to the existence of an $n$-virtual diagonal, an $(n-1)$-cocycle $D: C_{n-1}(A) \rightarrow C_{n+1}(A)^{* *}$ such that

$$
\pi_{n+1}^{* *}\left(D\left(a_{1} \otimes \cdots \otimes a_{n-1}\right)\right)=\pi_{n+1}\left(e \otimes a_{1} \otimes \cdots \otimes a_{n-1} \otimes e\right)
$$

for all $a_{i} \in A$. (The terminology used here is explained in §2.) A homological interpretation of this result and its non-unital counterpart is given in [17].

A very simple example of a 2 -amenable Banach algebra that is not amenable is the algebra $T_{2}$ of upper triangular complex matrices. (From the point of view of homological algebra, this is a consequence of a result of Selivanov. For a discussion of this see [17].)

A key problem is whether $n$-amenability $(n>1)$ is equivalent to amenability for $C^{*}$-algebras. The answer to this is (to the authors' knowledge) unknown. The 2-amenability of $T_{2}$ suggests that non-self-adjoint operator algebras, in particular triangular algebras, are natural algebras for investigating higher-dimensional amenability.

Before considering such algebras, we first look (in $\S 3$ ) at an incomplete result of [17]. It was shown there (Corollary 4.1) that if $n=2,3$ then $(n-1)$-amenability of $A$ is equivalent to the existence of a coboundary $n$-virtual diagonal. (The significance of this for the above problem of $n$-amenability for $C^{*}$-algebras is that if we could show, for example, that every 2 -amenable $C^{*}$-algebra admitted a coboundary 2 virtual diagonal, then 2-amenability would imply amenability (= nuclearity).) We show that this result is true for any $n \geq 2$.

In $\S 4$, we investigate higher-dimensional amenability for the algebras $T_{n}(n \geq 2)$ of upper triangular, complex $n \times n$ matrices. In [17], it was shown that for $n \geq 2$, $T_{n}$ is $(n-1)$-amenable. We will show that $T_{n}$ is 2 -amenable ${ }^{1}$ (but not amenable) by exhibiting explicitly a 2-virtual diagonal for $T_{n}$. We also show that certain other finite-dimensional algebras $B_{n}$, obtained by using the join and suspension operations of Gilfeather and Smith ([10], [9]), are $(n+1)$-amenable but not $n$ amenable. The problem of finding simple examples of this phenomenon was first raised in [13].

It is tempting to try to use the 2-virtual diagonals for the $T_{n}$ 's to construct a 2 -virtual diagonal for $T^{\infty}$, since the latter is a $w^{*}$-inductive limit of the $T_{n}$ 's. We show that this is not possible. More precisely, we show that if $\mathcal{N}$ is a complete nest of closed subspaces of a Hilbert space $H$ containing at least $n$ distinct non-zero elements, then $\|\delta\| \geq n-3$ for any 2-virtual diagonal on $\operatorname{Alg}(\mathcal{N})$. Thus there does not exist a uniformly bounded sequence of 2-virtual diagonals on the $T_{n}$ 's, and so it is impossible to obtain a 2-virtual diagonal on $T^{\infty}$ (or on any nest algebra whose nest has infinitely many elements) as a cluster point of such a sequence.

These abstract methods do not give simple examples of non-vanishing second cohomology; this is addressed in $\S 5$. In this section, we investigate the cohomology groups $H^{n}\left(T^{\infty}, J\right)$ where $J$ is a strong operator closed two-sided ideal in $T^{\infty}$. The groups $H^{1}\left(T^{\infty}, J\right)$ have been determined by Erdos and Power [7]. They also

${ }^{1}$ Y.V. Selivanov has informed the authors that he has also proved this result, using homological techniques. 
characterize the strong operator closed two-sided ideals in a general nest algebra using certain order homomorphisms. Using this result, we show that $J$ can be characterized as all those operators in $T^{\infty}$ whose matrices vanish on a certain part $V$ of $\mathbb{Z}^{+} \times \mathbb{Z}^{+}$. The complement $W$ of this part is built up out of triangular pieces. To investigate $H^{2}\left(T^{\infty}, J\right)$, it is helpful to use the long exact sequence property of $H^{n}\left(T^{\infty}, \cdot\right)$. For this purpose, it is important that $J$ be complemented in $T^{\infty}$ as a Banach space. For large classes of ideals this is the case, but it is not true in general. ${ }^{2}$ For many such ideals $J$ (including the case when $J$ is the ideal of strictly upper triangular operators) we show that $H^{n}\left(T^{\infty}, J\right)=0$ for $n \geq 2$. We also show that there are simple examples of ideals $J$ for which $H^{2}\left(T^{\infty}, J\right) \neq 0$.

We are grateful to David Larson for helpful discussions.

\section{Some PRELIminaries on BANACH ALGEBRA COHOMOlOgy}

Let $X$ be a Banach $A$-bimodule. We recall first how the cohomology groups $H^{n}(A, X)$ are defined. For each $n \geq 1$, let $C_{n}(A)$ be the $n$-fold projective tensor product $A \widehat{\otimes} \cdots \widehat{\otimes} A$ and let $C^{n}(A, X)$ be the Banach space of bounded $n$-linear maps from the $n$-fold Cartesian product $A \times \cdots \times A$ into $X$. Canonically, $C^{n}(A, X)=$ $B\left(C_{n}(A), X\right)$. The elements of $C^{n}(A, X)$ are called $n$-cochains. The bounded linear map $\delta^{n}$ (or simply $\delta$ ) from $C^{n}(A, X)$ into $C^{n+1}(A, X)$ is then defined by:

$$
\begin{aligned}
\delta^{n} f\left(a_{1}, \ldots, a_{n+1}\right)= & a_{1} f\left(a_{2}, \ldots, a_{n+1}\right) \\
& +\sum_{k=1}^{n}(-1)^{k} f\left(a_{1}, \ldots, a_{k-1}, a_{k} a_{k+1}, \ldots, a_{n+1}\right) \\
& +(-1)^{n+1} f\left(a_{1}, \ldots, a_{n}\right) a_{n+1} .
\end{aligned}
$$

The space ker $\delta^{n}$ is sometimes written $Z^{n}(A, X)$. The elements of $Z^{n}(A, X)=$ ker $\delta^{n}$ are called the $n$-cocycles and the elements of $\operatorname{Im} \delta^{n-1}=B^{n-1}(A, X)$ are called the $n$-coboundaries. Finally, for $n \geq 1$, we define

$$
H^{n}(A, X)=\operatorname{ker} \delta^{n} / \operatorname{Im} \delta^{n-1} .
$$

In the case $n=0$ we take $C^{0}(A, X)$ to be $X$, and $\delta^{0}: X \rightarrow C^{1}(A, X)$ is given by:

$$
\delta^{0}(x)(a)=a x-x a .
$$

We take $H^{0}(A, X)$ to be $\{x \in X: a x=x a$ for all $a \in A\}$.

A very useful result due to Kadison and Ringrose ([14], §4) enables us to replace an $n$-cocycle $T$ by an equivalent one for which elements of a certain kind of subalgebra $B$ of $A$ move in and out of the expression for $T$ as if they were scalars. (For precise recent statements, see [9], [17].) Thus $T$ is equivalent to a $B$-multimodular $n$-cocycle $T^{\prime}$, where the multimodular property means that for $a_{i} \in A, b \in B$ :

(a) $T^{\prime}\left(a_{1}, \ldots, a_{n}\right)=0$ whenever an $a_{i} \in B$;

(b) $b T^{\prime}\left(a_{1}, \ldots, a_{n}\right)=T^{\prime}\left(b a_{1}, a_{2}, \ldots, a_{n}\right), T^{\prime}\left(a_{1}, \ldots, a_{n}\right) b=T^{\prime}\left(a_{1}, \ldots, a_{n} b\right)$;

(c) $T^{\prime}\left(a_{1}, \ldots, a_{i} b, a_{i+1}, \ldots, a_{n}\right)=T^{\prime}\left(a_{1}, \ldots, a_{i}, b a_{i+1}, \ldots, a_{n}\right)$.

Of course, the notion of $B$-multimodularity applies to cochains in general. Multimodularity can be achieved for abelian $C^{*}$-algebras $B$, and this is sufficient for our purposes. In the case where we will require multimodular cocycles - when $A$ is a matrix algebra or $T^{\infty}$ - we will take $B$ to be the diagonal $C^{*}$-algebra.

\footnotetext{
${ }^{2}$ We are grateful to Ken Davidson for pointing this out to us.
} 
To understand the significance of the equality (1), we need to see its origins in Banach homology theory ([12]). In this theory, functors $\operatorname{Ext}_{A^{e}}^{n}(X, Y)$ are developed in a manner analogous to the corresponding functors in homological algebra. (Here $A^{e}=A \widehat{\otimes} A^{o p}$ and $X, Y$ are Banach $A$-bimodules.) The groups $H^{n}(A, X)$ are then just $\operatorname{Ext}_{A^{e}}^{n}(A, X)$, which can be calculated using the standard projective resolution for $A$ :

$$
0 \longleftarrow A \stackrel{\pi_{2}}{\longleftarrow} C_{2}(A) \stackrel{\pi_{3}}{\longleftarrow} C_{3}(A) \longleftarrow \cdots
$$

with $\pi_{n}: C_{n}(A) \rightarrow C_{n-1}(A)$ the morphism given by

$$
\pi_{n}\left(a_{1} \otimes \cdots \otimes a_{n}\right)=\sum_{i=1}^{n}(-1)^{i+1} a_{1} \otimes \cdots \otimes a_{i} a_{i+1} \otimes a_{i+1} \otimes \cdots \otimes a_{n} .
$$

(The morphism $\pi_{n}$ can be regarded as a "higher dimensional" product.) The sequence from which we obtain $\operatorname{Ext}_{A^{e}}^{n}(A, X)$ is given by applying $\operatorname{Hom}_{A^{e}}(\cdot, X)$ to $(2)$, and this is effectively the same as the sequence of $C^{n}(A, X)$ 's, connected by the $\delta$ 's.

Although in this paper we will use only the cocycle approach to $H^{n}(A, X)$, the preceding discussion explains why the $\pi_{n}$ maps in (3) play an important role in the theory. In particular, at a very direct level, we can conveniently rewrite the formula (1) for $\delta^{n}$ as

$$
\begin{aligned}
\delta^{n} f\left(a_{1} \otimes \cdots \otimes a_{n+1}\right) & =a_{1} f\left(a_{2} \otimes \cdots \otimes a_{n}\right)-f\left(\pi_{n+1}\left(a_{1} \otimes \cdots \otimes a_{n+1}\right)\right) \\
& +f\left(a_{1} \otimes \cdots \otimes a_{n}\right) a_{n+1} .
\end{aligned}
$$

As discussed earlier, $A$ is called $n$-amenable if $H^{n}\left(A, X^{*}\right)=0$ for all Banach $A$-bimodules $X$. We also stated the Effros-Kishimoto result in $\S 1$, that $n$ amenability is equivalent to the existence of an $n$-virtual diagonal, an $(n-1)$ cocycle $D: C_{n-1}(A) \rightarrow C_{n+1}(A)^{* *}$ satisfying (0). (The $\pi_{n+1}$ in (0) is, of course, the "higher dimensional" $\pi$-map defined as above.)

\section{When Does $n$-AMENABILITY IMPly $(n-1)$-AMENABiLity?}

It is well-known (e.g. [12], p. 254, [13], p. 9) that for $n \geq 2,(n-1)$-amenability implies $n$-amenability for the Banach algebra $A$. We will show that $A$ is $(n-1)$ amenable if and only if there exists an $n$-virtual diagonal that is a coboundary. (Of course, $n$-amenability is equivalent to the existence of an $n$-virtual diagonal, but such a cocycle will not, in general, be a coboundary.)

Theorem 3.1. For $n \geq 2$, the Banach algebra $A$ is $(n-1)$-amenable if and only if there exists an n-virtual diagonal for $A$ which is a coboundary.

Proof. By [17], Theorem 4.2, it is sufficient to exhibit a $Z \in C_{n-2}\left(A, C_{n+1}^{* *}\right)$ such that

$$
\pi_{n+1}^{* *}\left((\delta Z)(a)-e \otimes a_{1} \otimes \cdots \otimes a_{n-2} \otimes e \otimes a_{n-1}\right)=0
$$

for all $a=a_{1} \otimes \cdots \otimes a_{n-1}, a_{i} \in A$. Let

$$
Z\left(a_{1} \otimes \cdots \otimes a_{n-2}\right)=(-1)^{n+1} e \otimes a_{1} \otimes \cdots \otimes a_{n-2} \otimes e \otimes e .
$$

We show that (5) is satisfied. 
Indeed, using (4), if $w=a_{1} \otimes \cdots \otimes a_{n-1}, a_{i} \in A$, then

$$
\begin{aligned}
(-1)^{n+1} \delta Z(w)= & a_{1} Z\left(a_{2} \otimes \cdots \otimes a_{n-1}\right)-Z\left(\pi_{n-1}(w)\right) \\
& +(-1)^{n-1} Z\left(a_{1} \otimes \cdots \otimes a_{n-2}\right) a_{n-1} \\
= & w \otimes e \otimes e-e \otimes \pi_{n-1}(w) \otimes e \otimes e \\
& +(-1)^{n-1} e \otimes a_{1} \otimes \cdots \otimes a_{n-2} \otimes e \otimes a_{n-1} .
\end{aligned}
$$

Applying $\pi_{n+1}$ to both sides of (6), we see that (5) will follow once we have shown that

$$
\pi_{n+1}(w \otimes e \otimes e)=\pi_{n+1}\left(e \otimes \pi_{n-1}(w) \otimes e \otimes e\right) .
$$

The left-hand side of $(7)$ is just $\pi_{n-1}(w) \otimes e \otimes e$. For the right-hand side:

$$
\begin{aligned}
\pi_{n+1}\left(e \otimes \pi_{n-1}(w) \otimes e \otimes e\right) & =\pi_{n-1}(w) \otimes e \otimes e-e \otimes \pi_{n-2} \circ \pi_{n-1}(w) \otimes e \otimes e \\
& =\pi_{n-1}(w) \otimes e \otimes e
\end{aligned}
$$

as required, since $\pi_{n-2} \circ \pi_{n-1}=0$. This gives (7).

It would be interesting to know what the non-unital version of the preceding theorem is, and what its homological interpretation is.

\section{Higher-Dimensional amenability for $T_{n}$ And the Algebras $B_{n}$}

Recall that $T_{n}$ is the algebra of upper triangular $n \times n$ complex matrices.

Theorem 4.1. For $n \geq 2, T_{n}$ is 2-amenable, but not amenable.

Proof. The non-amenability of $T_{n}$ is proved in [17], Proposition 3.1.

To prove the 2-amenability of $T_{n}$, we exhibit a 2-virtual diagonal $D: T_{n} \rightarrow$ $T_{n} \widehat{\otimes} T_{n} \widehat{\otimes} T_{n}$. Let $e_{i j}(1 \leq i \leq j \leq n)$ be the canonical basis of matrix units for $T_{n}$ and define $D$ by:

$$
D\left(e_{i j}\right)=\sum_{s \neq j} e_{i j} \otimes e_{j j} \otimes e_{s s}-\sum_{t \neq i} e_{t t} \otimes e_{t t} \otimes e_{i j}+\sum_{p=i}^{j-1} e_{i p} \otimes e_{p p+1} \otimes e_{p+1 j} .
$$

The third sum of (8) is interpreted to be 0 if $i=j$.

To prove that $D$ as defined above is a 2 -virtual diagonal, we need to show that $D$ is a derivation on $T_{n}$ and that for all $i, j$,

$$
\pi_{3}\left(D\left(e_{i j}\right)\right)=e_{i j} \otimes 1-1 \otimes e_{i j} .
$$

We first prove that $D$ is a derivation. We need to show that for $1 \leq i \leq j \leq n$, $1 \leq k \leq \ell \leq n$, we have

$$
D\left(e_{i j} e_{k \ell}\right)=e_{i j} D\left(e_{k \ell}\right)+D\left(e_{i j}\right) e_{k \ell} .
$$


Now

$$
\begin{array}{r}
e_{i j} D\left(e_{k \ell}\right)+D\left(e_{i j}\right) e_{k \ell}=e_{i j} \\
\left(\sum_{s \neq \ell} e_{k \ell} \otimes e_{\ell \ell} \otimes e_{s s}-\sum_{t \neq k} e_{t t} \otimes e_{t t} \otimes e_{k \ell}\right. \\
\left.+\sum_{p=k}^{\ell-1} e_{k p} \otimes e_{p p+1} \otimes e_{p+1 \ell}\right) \\
+\left(\sum_{s \neq j} e_{i j} \otimes e_{j j} \otimes e_{s s}-\sum_{t \neq i} e_{t t} \otimes e_{t t} \otimes e_{i j}\right. \\
\left.+\sum_{p=i}^{j-1} e_{i p} \otimes e_{p p+1} \otimes e_{p+1 j}\right) e_{k \ell .}
\end{array}
$$

Suppose that $j \neq k$. Then (11) gives

$$
e_{i j} D\left(e_{k \ell}\right)+D\left(e_{i j}\right) e_{k \ell}=-e_{i j} \otimes e_{j j} \otimes e_{k \ell}+e_{i j} \otimes e_{j j} \otimes e_{k \ell}=0
$$

and (10) follows since its left-hand side is $D(0)$.

Suppose, then, that $j=k$. We will assume that $i<j<\ell$, the cases where $i=j$ or $j=\ell$ being dealt with similarly. Then, from (11),

$$
\begin{aligned}
& e_{i j} D\left(e_{j \ell}\right)+D\left(e_{i j}\right) e_{j \ell} \\
&=\left(\sum_{s \neq \ell} e_{i \ell} \otimes e_{\ell \ell} \otimes e_{s s}+\sum_{p=j}^{\ell-1} e_{i p} \otimes e_{p p+1} \otimes e_{p+1 \ell}\right) \\
&+\left(-\sum_{t \neq i} e_{t t} \otimes e_{t t} \otimes e_{i \ell}+\sum_{p=i}^{j-1} e_{i p} \otimes e_{p p+1} \otimes e_{p+1 \ell}\right) \\
&= \sum_{s \neq \ell} e_{i \ell} \otimes e_{\ell \ell} \otimes e_{s s}-\sum_{t \neq i} e_{t t} \otimes e_{t t} \otimes e_{i \ell}+\sum_{p=i}^{\ell-1} e_{i p} \otimes e_{p p+1} \otimes e_{p+1 \ell} \\
&= D\left(e_{i \ell}\right)=D\left(e_{i j} e_{j \ell}\right) .
\end{aligned}
$$

Thus $D$ is a derivation.

To prove $(9)$

$$
\begin{aligned}
\pi_{3}\left(D\left(e_{i j}\right)\right)= & \sum_{s \neq j}\left(e_{i j} \otimes e_{s s}-0\right)-\sum_{t \neq i}\left(e_{t t} \otimes e_{i j}-0\right) \\
& \quad+\sum_{p=i}^{j-1}\left(e_{i p+1} \otimes e_{p+1 j}-e_{i p} \otimes e_{p j}\right) \\
= & e_{i j} \otimes\left(1-e_{j j}\right)-\left(1-e_{i i}\right) \otimes e_{i j}+\left(e_{i j} \otimes e_{j j}-e_{i i} \otimes e_{i j}\right) \\
= & e_{i j} \otimes 1-1 \otimes e_{i j}
\end{aligned}
$$

where we have used the facts that $\sum_{s=1}^{n} e_{s s}=1$ and that the non-extreme terms in the " $\sum_{p=i}^{j-1}$ " expression cancel in pairs. 
Other examples of $n$-amenability are provided by using joins and suspensions of Gilfeather and Smith ([10], [9]).

If $H$ and $K$ are Hilbert spaces and $A$ and $B$ are norm closed unital subalgebras of $B(H)$ and $B(K)$ respectively, then the join $A \# B$ is the subalgebra of $B(K \oplus H)$ whose elements are of the form

$$
\left[\begin{array}{ll}
b & 0 \\
u & a
\end{array}\right]
$$

with $a \in A, b \in B$ and $u \in B(K, H)$. Let $D_{2}$ be the diagonal algebra of $2 \times 2$ complex matrices $\left(\cong \mathbb{C}^{2}\right)$, and let $A_{4}$ be $D_{2} \# D_{2}$. The elements of $A_{4}$ are those $4 \times 4$ complex matrices of the form

$$
\left[\begin{array}{llll}
* & 0 & 0 & 0 \\
0 & * & 0 & 0 \\
* & * & * & 0 \\
* & * & 0 & *
\end{array}\right]
$$

where $*$ denotes an arbitrary scalar.

The two-point suspension $S(A)$ of the algebra $A$ is the subalgebra of $B\left(\mathbb{C}^{2} \oplus H\right)$ whose elements are of the form

$$
\left[\begin{array}{ll}
d & 0 \\
u & a
\end{array}\right]
$$

where $d \in D_{2}, a \in A$ and $u \in B\left(\mathbb{C}^{2}, H\right)$. We define

$$
B_{n}=S^{n-1}\left(A_{4}\right)
$$

where $S^{n-1}$ means that the suspension operation is applied recursively $(n-1)$ times. It is easily checked that $B_{n} \subseteq M_{2(n-1)+4}$. Of course, $B_{1}=A_{4}$.

Theorem 4.2. The algebra $B_{n}$ is $(n+1)$-amenable but not $n$-amenable.

Proof. We prove first, by induction on $n$, that if $m>n$ and $e_{i_{r} i_{r+1}} \in B_{n}(1 \leq r \leq$ $m$ ) then at least one of the $e_{i_{r} i_{r+1}}$ 's is of the form $e_{a a}$. Suppose firstly that $n=1$ so that $B_{n}=A_{4}$. Suppose that the $e_{i_{r} i_{r+1}}(1 \leq r \leq m)$ are such that none is of the form $e_{a a}$. From (12), the $i_{r} i_{r+1}$ 's must come from 31, 32, 41 and 42 . But this is impossible since all of the latter end in 1 or 2 while none starts with 1 or 2 and $m \geq 2$.

Suppose that the result is true now for some $n$ and let $m>n+1$. Suppose that there exist $e_{i_{r} i_{r+1}} \in B_{n+1}(1 \leq r \leq n+1)$ with no $e_{i_{r} i_{r+1}}$ of the form $e_{a a}$. From (13), we have, for each $r$, either $e_{i_{r} i_{r+1}} \in B_{n}$ or $2 \leq i_{r} \leq 2 n+2,1 \leq i_{r+1} \leq 2$. If $2 \leq i_{r} \leq 2 n+2,1 \leq i \leq 2$, then $e_{i_{r} i_{r+1}}$ cannot be followed by $e_{i_{r+1} i_{r+2}} \in B_{n}$ since it is in one or other of the top two rows of $M_{2 n+2}$. Thus at most the last element $e_{i_{m} i_{m+1}}$ is not in $B_{n}$. We conclude that $e_{i_{1} i_{2}}, \ldots, e_{i_{m-1} i_{m}} \in B_{n}$, and since $n<m-1$, the induction hypothesis gives $i_{r}=i_{r+1}$ for some $r$. This concludes the induction proof.

Let $n \geq 1, m>n$, and let $X$ be a Banach $B_{n}$-bimodule. We prove that $H^{m}\left(B_{n}, X\right)=0$. (This is equivalent to $(n+1)$-amenability.) It is sufficient to show that $f \in Z^{m}\left(B_{n}, X\right)$ is a coboundary. We can suppose that $f$ is multimodular for the diagonal of $M_{2 n+2}$. We show that $f=0$. To this end, it is sufficient to show that

$$
f\left(e_{i_{1} j_{1}}, \ldots, e_{i_{m} j_{n}}\right)=0 .
$$


Writing $e_{i_{r} j_{r}}=e_{i_{r} j_{r}} e_{j_{r} j_{r}}$ and passing the $e_{j_{r} j_{r}}$ over to left-multiply the adjacent term $e_{i_{r+1} j_{r+1}}$, we can suppose that $j_{r}=i_{r+1}$. By the result whose proof was given earlier, at least one of the $e_{i_{r} i_{r+1}}$ 's is an $e_{a a}$. Since $f$ is multimodular, (14) follows. Thus $B_{n}$ is $(n+1)$-amenable. To prove that it is not $n$-amenable, it is sufficient to show that $H^{n}\left(B_{n}, M_{2 n+2}\right) \neq 0$, where $M_{2 n+2}$ is regarded as a $B_{n}$-bimodule using the natural multiplication.

Suppose firstly that $n=1$. Then $B_{1}=A_{4}=D_{2} \# D_{2}$, and by [10], Theorem 3.3,

$$
H^{1}\left(A_{4}, M_{4}\right)=H^{0}\left(D_{2}\right) \otimes H^{0}\left(D_{2}\right)=\mathbb{C} \otimes \mathbb{C}=\mathbb{C} \neq 0
$$

(since $H^{0}\left(D_{2}\right)=D_{2} / \mathbb{C}=\mathbb{C}$ ). Suppose, then, that $n>1$. Now Theorem 3.6 of $[9]$ gives, for a unital algebra $A \subseteq B(H)$, the formula

$$
H^{n-1}(A, B(H))=H^{n}\left(S(A), B\left(\mathbb{C}^{2} \oplus H\right)\right) .
$$

We apply this recursively to the relation $B_{n}=S\left(B_{n-1}\right)$ to obtain

$$
H^{n}\left(B_{n}, M_{2 n+2}\right)=H^{n-1}\left(B_{n-1}, M_{2 n}\right)=\cdots=H^{2}\left(B_{2}, M_{6}\right)=H^{1}\left(B_{1}, M_{4}\right)=\mathbb{C} .
$$

Thus $B_{n}$ is not $n$-amenable.

We saw, at the beginning of this section that every $T_{n}$ is 2 -amenable. If we can find a uniformly bounded sequence $\left\{\delta_{n}\right\}$, where $\delta_{n}$ is a 2-virtual diagonal on $T_{n}$, then we could take an appropriate weak* cluster point of $\left\{\delta_{n}\right\}$ to obtain a 2virtual diagonal on the weak* inductive limit $T^{\infty}$ of the $T_{n}$ 's. (This parallels the production of a virtual diagonal on an AFD von Neumann algebra from virtual diagonals on its finite-dimensional $C^{*}$-subalgebras.) However, we will now obtain a norm estimate for 2-virtual diagonals on nest algebras which, in particular, shows that no nest algebra for an infinite nest can be 2 -amenable. The calculations below are notationally complicated, largely because we must work not with $A \widehat{\otimes} A \widehat{\otimes} A$ but with its second dual. For example, the operation of left multiplication by $x$ in the middle variable is simply expressed by

$$
a \otimes b \otimes c \rightarrow a \otimes x b \otimes c .
$$

To express the same operation on the second dual we must define a map $\lambda: A \rightarrow A$, given by left multiplication by $x$, and then consider

$$
(I \otimes \lambda \otimes I)^{* *}(u), \quad u \in(A \widehat{\otimes} A \widehat{\otimes} A)^{* *} .
$$

The reader may find it enlightening to assume that the 2 -virtual diagonals below map into $A \widehat{\otimes} A \widehat{\otimes} A$, when the more transparent forms of various operations are available.

Let $\mathcal{N}$ be a complete nest of subspaces of a Hilbert space $H$, and suppose that $\mathcal{N}$ contains at least $n$ distinct non-zero subspaces $N_{1} \subseteq N_{2} \subseteq \cdots \subseteq N_{n}=H$. Denote by $A$ the algebra

$$
\{t: t N \subseteq N, N \in \mathcal{N}\}
$$

and let $p_{i}$ be the projection onto $N_{i}, 1 \leq i \leq n$. Then define $e_{11}=p_{1}$, while $e_{i i}=p_{i}-p_{i-1}$ for $i \geq 2$. Clearly $\left\{e_{i i}: 1 \leq i \leq n\right\} \subseteq A$ is a set of orthogonal projections whose sum is 1 . Now choose unit vectors $\xi_{1} \in N_{1}, \xi_{i} \in N_{i} \ominus N_{i-1}$, $2 \leq i \leq n$, and denote by $e_{i j}(1 \leq i<j \leq n)$ the rank one operator $\xi_{i} \otimes \xi_{j}$ defined by

$$
e_{i j}(\xi)=\left\langle\xi, \xi_{j}\right\rangle \xi_{i}, \quad \xi \in H
$$


If $p$ is a projection in $A$ corresponding to an element of the nest, then, for a given pair $i<j$, there are three possibilities:

(a) $p \leq p_{i} \leq p_{j}$,

(b) $p_{i} \leq p \leq p_{j}$

(c) $p_{i} \leq p_{j} \leq p$.

Since $p^{\perp} e_{i j} p=\left(p^{\perp} \xi_{i}\right) \otimes\left(p \xi_{j}\right)$, we see that $p^{\perp} e_{i j} p=0$ in all three cases: in the first $p \xi_{j}=0$ while in the second and third $p^{\perp} \xi_{i}=0$. It follows that $\left\{e_{i j}: 1 \leq i \leq\right.$ $j \leq n\} \subseteq A$. Moreover, the relations

$$
e_{i j} e_{k \ell}=\left\{\begin{array}{cc}
0, & j \neq k, \\
e_{i \ell}, & j=k,
\end{array}\right.
$$

for $i \leq j, k \leq \ell$ are clear from the definitions. Thus the set $\left\{e_{i j}: 1 \leq i \leq j \leq n\right\}$ acts as a set of upper triangular matrix units, although it should be noted that $e_{i j}$ is rank one in general only if $i<j$.

We now introduce some auxiliary operators which play a role in the last theorem of this section. There are many relationships between them; the ones we will need are set out in the proposition below.

For $1 \leq i \leq n$, define $\rho_{i}, \lambda_{i}: A \rightarrow A$ to be respectively right and left multiplication by $e_{i i}$, and let $m: A \widehat{\otimes} A \widehat{\otimes} A \rightarrow A$ be given by

$$
m(a \otimes b \otimes c)=a b c .
$$

We write $U$ for the compact unitary group of the abelian $C^{*}$-algebra generated by $\left\{e_{11}, \ldots, e_{n n}\right\}$. The typical element of $U$ has the form $\sum_{j=1}^{n} e^{i \theta_{j}} e_{j j}$, and Haar measure $d \mu$ on $U$ is the $n$-fold normalized Lebesgue measure $\frac{1}{(2 \pi)^{n}} d \theta_{1} \ldots d \theta_{n}$ on the $n$-torus $\mathbb{T}^{n}$. We then define $\xi_{2}: A \widehat{\otimes} A \rightarrow A \widehat{\otimes} A$ and $\xi_{3}: A \widehat{\otimes} A \widehat{\otimes} A \rightarrow A \widehat{\otimes} A \widehat{\otimes} A$ by

$$
\begin{aligned}
\xi_{2}(a \otimes b) & =\int_{U} a u^{*} \otimes u b d \mu(u), \\
\xi_{3}(a \otimes b \otimes c) & =\int_{U \times U} \int_{\cup} a u^{*} \otimes u b v^{*} \otimes v c d \mu(u) d \mu(v) .
\end{aligned}
$$

We will also need $\mu_{1}, \mu_{2}: A \widehat{\otimes} A \widehat{\otimes} A \rightarrow A \widehat{\otimes} A$, defined by

$$
\begin{aligned}
& \mu_{1}(a \otimes b \otimes c)=a b \otimes c, \\
& \mu_{2}(a \otimes b \otimes c)=a \otimes b c .
\end{aligned}
$$

Finally, we denote the contractive projection $\sum_{i=1}^{n-1} \lambda_{i} \rho_{i+1}$ of $A$ onto $\bigoplus_{i=1}^{n-1} e_{i i} A e_{i+1 i+1}$ by $\alpha$.

Proposition 4.3. The following properties hold:

(i) $\left\{\lambda_{1}, \ldots, \lambda_{n}, \rho_{1}, \ldots, \rho_{n}\right\}$ is a commuting set of maps,

$$
\lambda_{i} \lambda_{j}=\rho_{i} \rho_{j}=0
$$

for $i \neq j$, and

$$
\lambda_{i} \rho_{j}=0
$$

for $i>j$;

$$
\|m\|=\left\|\xi_{2}\right\|=\left\|\xi_{3}\right\|=1 ;
$$




$$
\begin{aligned}
& m=\pi_{2} \mu_{1} \\
& \pi_{3}=\mu_{1}-\mu_{2} \\
& \xi_{2}=\sum_{i=1}^{n} \rho_{i} \otimes \lambda_{i} ; \\
& \xi_{3}=\sum_{1 \leq i \leq j \leq n} \rho_{i} \otimes \lambda_{i} \rho_{j} \otimes \lambda_{j} ; \\
& \pi_{3} \xi_{3}=\xi_{2} \pi_{3} \\
& \left(\lambda_{i} \otimes I \otimes \rho_{i+1}\right) \xi_{3}=\lambda_{i} \rho_{i} \otimes \lambda_{i} \rho_{i} \otimes \lambda_{i} \rho_{i+1}+\lambda_{i} \rho_{i} \otimes \lambda_{i} \rho_{i+1} \otimes \lambda_{i+1} \rho_{i+1} \\
& +\lambda_{i} \rho_{i+1} \otimes \lambda_{i+1} \rho_{i+1} \otimes \lambda_{i+1} \rho_{i+1} \\
& \pi_{2}\left(\rho_{i+1} \otimes \lambda_{i+1}\right) \pi_{3}\left(\lambda_{i} \otimes I \otimes \rho_{i+1}\right) \xi_{3} \\
& =\pi_{2}\left(\rho_{i+1} \otimes \lambda_{i+1}\right) \pi_{3}\left(\lambda_{i} \otimes I \otimes \rho_{i+1}\right)(I \otimes \alpha \otimes I) \xi_{3} \\
& =\pi_{2}\left(\rho_{i+1} \otimes \lambda_{i+1}\right) \mu_{1}\left(\lambda_{i} \otimes I \otimes \rho_{i+1}\right)(I \otimes \alpha \otimes I) \xi_{3} ; \\
& \pi_{2} \mu_{1}\left(\lambda_{i} \otimes I \otimes \rho_{i+1}\right)(I \otimes \alpha \otimes I) \xi_{3} \\
& \pi_{2}\left(\rho_{i+1} \otimes \lambda_{i+1}\right) \mu_{1}\left(\lambda_{i} \otimes I \otimes \rho_{i+1}\right)(I \otimes \alpha \otimes I) \xi_{3} .
\end{aligned}
$$

Proof. (i) The first two statements are clear from the definitions. If $i>j$, then

$$
\lambda_{i} \rho_{j}(a)=e_{i i} a e_{j j}=e_{i i} p_{j}^{\perp} a p_{j} e_{j j}
$$

since $p_{j} e_{j j}=e_{j j}$ and $e_{i i} p_{j}^{\perp}=e_{i i}$. However, $p_{j}^{\perp} a p_{j}=0$ for $a \in A$, so $\lambda_{i} \rho_{j}=0$.

(ii) It is clear, from the definition of the norm, that $m, \xi_{2}$ and $\xi_{3}$ are contractions. Since $m(1 \otimes 1 \otimes 1)=1$, it follows that $\|m\|=1$. Now

$$
\begin{aligned}
m \xi_{3}(1 \otimes 1 \otimes 1) & =m\left(\int_{U \times U} u^{*} \otimes u v^{*} \otimes v d \mu(u) d \mu(v)\right) \\
& =\iint_{U \times U} 1 d \mu(u) d \mu(v) \\
& =1
\end{aligned}
$$

and so $\left\|\xi_{3}\right\|=1$. A similar calculation gives $\pi_{2} \xi_{2}(1 \otimes 1)=1$, and thus $\left\|\xi_{2}\right\|=1$.

(iii) Since $\pi_{2} \mu_{1}(a \otimes b \otimes c)=\pi_{2}(a b \otimes c)=a b c$, we have $m=\pi_{2} \mu_{1}$.

(iv) This is clear from the definitions.

(v) For $a, b \in A$,

$$
\begin{aligned}
\xi_{2}(a \otimes b) & =\frac{1}{(2 \pi)^{n}} \int_{0}^{2 \pi} \cdots \int_{0}^{2 \pi} a \sum_{j=1}^{n} e^{i \theta_{j}} e_{j j} \otimes \sum_{k=1}^{n} e^{-i \theta_{k}} e_{k k} b d \theta_{1} \ldots d \theta_{n} \\
& =\frac{1}{(2 \pi)^{n}} \int_{0}^{2 \pi} \cdots \int_{0}^{2 \pi} \sum_{j, k=1}^{n} e^{i\left(\theta_{j}-\theta_{k}\right)} a e_{j j} \otimes e_{k k} b d \theta_{1} \ldots d \theta_{n} \\
& =\sum_{j=1}^{n} a e_{j j} \otimes e_{j j} b,
\end{aligned}
$$

since the terms with $j \neq k$ vanish after integration. Thus $\xi_{2}=\sum_{i=1}^{n} \rho_{i} \otimes \lambda_{i}$. 
(vi) Integration, as in (v), establishes that

$$
\xi_{3}(a \otimes b \otimes c)=\sum_{i, j=1}^{n} a e_{i i} \otimes e_{i i} b e_{j j} \otimes e_{j j} c,
$$

and so

$$
\xi_{3}=\sum_{i, j=1}^{n} \rho_{i} \otimes \lambda_{i} \rho_{j} \otimes \lambda_{j} .
$$

From (i) the terms with $i>j$ vanish, and it follows that

$$
\xi_{3}=\sum_{1 \leq i \leq j \leq n} \rho_{i} \otimes \lambda_{i} \rho_{j} \otimes \lambda_{j} .
$$

(vii) From (vi),

$$
\begin{aligned}
\pi_{3} \xi_{3}(a \otimes b \otimes c) & =\pi_{3}\left(\sum_{i, j=1}^{n} a e_{i i} \otimes e_{i i} b e_{j j} \otimes e_{j j} c\right) \\
& =\sum_{i, j=1}^{n} a e_{i i} b e_{j j} \otimes e_{j j} c-\sum_{i, j=1}^{n} a e_{i i} \otimes e_{i i} b e_{j j} c \\
& =\sum_{j=1}^{n} a b e_{j j} \otimes e_{j j} c-\sum_{i=1}^{n} a e_{i i} \otimes e_{i i} b c
\end{aligned}
$$

From (v),

$$
\begin{aligned}
\xi_{2} \pi_{3}(a \otimes b \otimes c) & =\xi_{2}(a b \otimes c-a \otimes b c) \\
& =\sum_{i=1}^{n}\left(a b e_{i i} \otimes e_{i i} c-a e_{i i} \otimes e_{i i} b c\right),
\end{aligned}
$$

and thus $\pi_{3} \xi_{3}=\xi_{2} \pi_{3}$.

(viii) From (vi),

$$
\begin{aligned}
\left(\lambda_{i} \otimes I \otimes \rho_{i+1}\right) \xi_{3} & =\lambda_{i} \otimes I \otimes \rho_{i+1}\left(\sum_{1 \leq j \leq k \leq n} \rho_{j} \otimes \lambda_{j} \rho_{k} \otimes \lambda_{k}\right) \\
& =\sum_{1 \leq j \leq k \leq n} \lambda_{i} \rho_{j} \otimes \lambda_{j} \rho_{k} \otimes \rho_{i+1} \lambda_{k} .
\end{aligned}
$$

By (i), terms for which $i>j$ or $k>i+1$ vanish from this sum, leaving

$$
\left(\lambda_{i} \otimes I \otimes \rho_{i+1}\right) \xi_{3}=\sum_{i \leq j \leq k \leq i+1} \lambda_{i} \rho_{j} \otimes \lambda_{j} \rho_{k} \otimes \rho_{i+1} \lambda_{k} .
$$

(ix) From (viii),

$$
\begin{aligned}
& \pi_{2}\left(\rho_{i+1} \otimes \lambda_{i+1}\right) \pi_{3}\left(\lambda_{i} \otimes I \otimes \rho_{i+1}\right) \xi_{3}(a \otimes b \otimes c) \\
&= \pi_{2}\left(\rho_{i+1} \otimes \lambda_{i+1}\right) \pi_{3}\left(e_{i i} a e_{i i} \otimes e_{i i} b e_{i i} \otimes e_{i i} c e_{i+1 i+1}\right. \\
& \quad+e_{i i} a e_{i i} \otimes e_{i i} b e_{i+1 i+1} \otimes e_{i+1 i+1} c e_{i+1 i+1} \\
&\left.+e_{i i} a e_{i+1 i+1} \otimes e_{i+1 i+1} b e_{i+1 i+1} \otimes e_{i+1 i+1} c e_{i+1 i+1}\right) .
\end{aligned}
$$

After applying $\pi_{3}$ to the first term in this sum, we obtain an element in

$$
\operatorname{span}\left\{x e_{i i} \otimes y, z \otimes e_{i i} w: x, y, z, w \in A\right\}
$$


which is then annihilated by $\rho_{i+1} \otimes \lambda_{i+1}$. Similarly, $\pi_{3}$ maps the third term into the range of the projection $\rho_{i+1} \otimes \lambda_{i+1}$ and is thus annihilated by $\pi_{2}\left(\rho_{i+1} \otimes \lambda_{i+1}\right)$ since $\pi_{2} \pi_{3}=0$. It follows that only the second term in (31) is significant, and so

$$
\begin{aligned}
& \pi_{2}\left(\rho_{i+1} \otimes \lambda_{i+1}\right) \pi_{3}\left(\lambda_{i} \otimes I \otimes \rho_{i+1}\right) \xi_{3}(a \otimes b \otimes c) \\
& \quad=\pi_{2}\left(\rho_{i+1} \otimes \lambda_{i+1}\right) \pi_{3}\left(e_{i i} a e_{i i} \otimes e_{i i} b e_{i+1 i+1} \otimes e_{i+1 i+1} c e_{i+1 i+1}\right) \\
& \quad=\pi_{2}\left(e_{i i} a e_{i i} b e_{i+1 i+1} \otimes e_{i+1 i+1} c e_{i+1 i+1}\right) \\
& \quad=e_{i i} a e_{i i} b e_{i+1 i+1} c e_{i+1 i+1} .
\end{aligned}
$$

Now $I \otimes \alpha \otimes I$ (which commutes with $\lambda_{i} \otimes I \otimes \rho_{i+1}$ ) annihilates any tensor whose middle term is $e_{k k} b e_{k k}$ while acting as the identity on any tensor whose middle term is $e_{i i} b e_{i+1 i+1}$, so by inserting this operator into the right hand side of (31) we obtain

$$
\begin{aligned}
& \pi_{2}\left(\rho_{i+1} \otimes \lambda_{i+1}\right) \pi_{3}\left(\lambda_{i} \otimes I \otimes \rho_{i+1}\right)(I \otimes \alpha \otimes I) \xi_{3}(a \otimes b \otimes c) \\
& \quad=\pi_{2}\left(\rho_{i+1} \otimes \lambda_{i+1}\right) \pi_{3}\left(e_{i i} a e_{i i} \otimes e_{i i} b e_{i+1 i+1} \otimes e_{i+1 i+1} c e_{i+1 i+1}\right) .
\end{aligned}
$$

It follows from (32) that

$$
\begin{aligned}
& \quad \pi_{2}\left(\rho_{i+1} \otimes \lambda_{i+1}\right) \pi_{3}\left(\lambda_{i} \otimes I \otimes \rho_{i+1}\right) \xi_{3} \\
& \quad=\pi_{2}\left(\rho_{i+1} \otimes \lambda_{i+1}\right) \pi_{3}\left(\lambda_{i} \otimes I \otimes \rho_{i+1}\right)(I \otimes \alpha \otimes I) \xi_{3} .
\end{aligned}
$$

Since

$$
\begin{aligned}
& \left(\rho_{i+1} \otimes \lambda_{i+1}\right) \mu_{2}\left(e_{i i} a e_{i i} \otimes e_{i i} b e_{i+1 i+1} \otimes e_{i+1 i+1} c e_{i+1 i+1}\right) \\
& \quad=\left(\rho_{i+1} \otimes \lambda_{i+1}\right)\left(e_{i i} a e_{i i} \otimes e_{i i} b e_{i+1 i+1} c e_{i+1 i+1}\right) \\
& \quad=0
\end{aligned}
$$

we may replace $\pi_{3}$ by $\mu_{1}-\mu_{2}$ in the right hand side of (33) to obtain that both terms in (34) are also equal to $\pi_{2}\left(\rho_{i+1} \otimes \lambda_{i+1}\right) \mu_{1}\left(\lambda_{i} \otimes I \otimes \rho_{i+1}\right)(I \otimes \alpha \otimes I) \xi_{3}$.

(x) To establish this part, it suffices to show that $\rho_{i+1} \otimes \lambda_{i+1}$ acts as the identity on terms of the form

$$
\mu_{1}\left(\lambda_{i} \otimes I \otimes \rho_{i+1}\right)(I \otimes \alpha \otimes I) \xi_{3}(a \otimes b \otimes c) .
$$

Using the right hand side of (32), it is clear that

$$
\left(\lambda_{i} \otimes I \otimes \rho_{i+1}\right)(I \otimes \alpha \otimes I) \xi_{3}(a \otimes b \otimes c)=e_{i i} a e_{i i} \otimes e_{i i} b e_{i+1 i+1} \otimes e_{i+1 i+1} c e_{i+1 i+1},
$$

and so

$$
\mu_{1}\left(\lambda_{i} \otimes I \otimes \rho_{i+1}\right)(I \otimes \alpha \otimes I) \xi_{3}(a \otimes b \otimes c)
$$

has the form $x e_{i+1 i+1} \otimes e_{i+1 i+1} y$. Clearly $\rho_{i+1} \otimes \lambda_{i+1}$ acts as the identity on any such element.

Theorem 4.4. Let $\mathcal{N}$ be a complete nest of closed subspaces of a Hilbert space and suppose that $\mathcal{N}$ has at least $n$ distinct elements. If $\delta$ is a 2-virtual diagonal for $A=\operatorname{Alg} \mathcal{N}$, then

$$
\|\delta\| \geq n-3
$$

In particular, this inequality holds for any 2-virtual diagonal for $T_{n}$, while no nest algebra arising from an infinite nest can have a 2-virtual diagonal. 
Proof. Let $\delta: A \rightarrow(A \widehat{\otimes} A \widehat{\otimes} A)^{* *}$ be a 2-virtual diagonal, so that

$$
\pi_{3}^{* *}(\delta(a))=a \otimes 1-1 \otimes a .
$$

Let $t$ be the element $\int_{U} u^{*} \otimes u \otimes 1 d \mu(u)$ of $A \widehat{\otimes} A \widehat{\otimes} A$. It is clear from the definition that $\|t\| \leq 1$, while integration, as in the preceding proposition, shows that

$$
t=\sum_{j=1}^{n} e_{j j} \otimes e_{j j} \otimes 1 .
$$

Then

$$
e_{r s} t-t e_{r s}=e_{r s} \otimes e_{s s} \otimes 1-\sum_{j=1}^{n} e_{j j} \otimes e_{j j} \otimes e_{r s}
$$

and so

$$
\begin{aligned}
\pi_{3}\left(e_{r s} t-t e_{r s}\right) & =e_{r s} \otimes 1-e_{r s} \otimes e_{s s}-\sum_{j=1}^{n} e_{j j} \otimes e_{r s}+e_{r r} \otimes e_{r s} \\
& =e_{r s} \otimes 1-e_{r s} \otimes e_{s s}-1 \otimes e_{r s}+e_{r r} \otimes e_{r s} \\
& =\pi_{3}^{* *}\left(\delta\left(e_{r s}\right)\right)-e_{r s} \otimes e_{s s}+e_{r r} \otimes e_{r s} .
\end{aligned}
$$

Let $\varepsilon: A \rightarrow(A \widehat{\otimes} A \widehat{\otimes} A)^{* *}$ be the derivation

$$
\varepsilon(a)=\delta(a)-(a t-t a), \quad a \in A .
$$

Then $\|\varepsilon\| \leq\|\delta\|+2$, and

$$
\pi_{3}^{* *}\left(\varepsilon\left(e_{r s}\right)\right)=e_{r s} \otimes e_{s s}-e_{r r} \otimes e_{r s}
$$

from (36). Now $\xi_{3}: A \widehat{\otimes} A \widehat{\otimes} A \rightarrow A \widehat{\otimes} A \widehat{\otimes} A$ is an $A$-bimodule map, so

$$
\gamma=\xi_{3}^{* *} \varepsilon: A \rightarrow(A \widehat{\otimes} A \widehat{\otimes} A)^{* *}
$$

is a derivation. From (22),

$$
\|\gamma\| \leq\|\varepsilon\| \leq\|\delta\|+2
$$

Using (25), (27), and (37), we see that

$$
\begin{aligned}
\pi_{3}^{* *}\left(\gamma\left(e_{r s}\right)\right) & =\pi_{3}^{* *} \xi_{3}^{* *} \varepsilon\left(e_{r s}\right) \\
& =\xi_{2}^{* *} \pi_{3}^{* *} \varepsilon\left(e_{r s}\right) \\
& =\xi_{2}\left(e_{r s} \otimes e_{s s}-e_{r r} \otimes e_{r s}\right) \\
& =\sum_{i=1}^{n}\left(\rho_{i} \otimes \lambda_{i}\right)\left(e_{r s} \otimes e_{s s}-e_{r r} \otimes e_{r s}\right) \\
& =e_{r s} \otimes e_{s s}-e_{r r} \otimes e_{r s} .
\end{aligned}
$$

Since $\pi_{3}$ is also an $A$-bimodule map, this leads to

$$
\pi_{3}^{* *}\left(e_{i i} \gamma\left(e_{i i+1}\right) e_{i+1 i+1}\right)=e_{i i+1} \otimes e_{i+1 i+1}-e_{i i} \otimes e_{i i+1} .
$$


Define $\beta: A \rightarrow(A \widehat{\otimes} A \widehat{\otimes} A)^{* *}$ by $\beta=(I \otimes \alpha \otimes I)^{* *} \gamma$. The $A$-bimodularity of $I \otimes \alpha \otimes I$ shows that $\beta$ is also a derivation. Now

$$
\begin{aligned}
& \pi_{2}^{* *}\left(\rho_{i+1} \otimes \lambda_{i+1}\right)^{* *} \mu_{1}^{* *}\left(e_{i i} \beta\left(e_{i i+1}\right) e_{i+1 i+1}\right) \\
& \quad=\pi_{2}^{* *}\left(\rho_{i+1} \otimes \lambda_{i+1}\right)^{* *} \mu_{1}^{* *}\left(\lambda_{i} \otimes I \otimes \rho_{i+1}\right)^{* *}(I \otimes \alpha \otimes I)^{* *} \gamma\left(e_{i i+1}\right) \\
& \quad=\pi_{2}^{* *}\left(\rho_{i+1} \otimes \lambda_{i+1}\right)^{* *} \pi_{3}^{* *}\left(\lambda_{i} \otimes I \otimes \rho_{i+1}\right)^{* *} \gamma\left(e_{i i+1}\right)
\end{aligned}
$$

from $(29)$

$$
\begin{aligned}
& =\pi_{2}^{* *}\left(\rho_{i+1} \otimes \lambda_{i+1}\right)^{* *} \pi_{3}^{* *}\left(e_{i i} \gamma\left(e_{i i+1}\right) e_{i+1 i+1}\right) \\
& =\pi_{2}^{* *}\left(\rho_{i+1} \otimes \lambda_{i+1}\right)^{* *}\left(e_{i i+1} \otimes e_{i+1 i+1}-e_{i i} \otimes e_{i i+1}\right)
\end{aligned}
$$

from $(38)$

$$
\begin{aligned}
& =\pi_{2}\left(e_{i i+1} \otimes e_{i+1 i+1}\right) \\
& =e_{i i+1} .
\end{aligned}
$$

It follows, using (30), that

$$
\pi_{2}^{* *} \mu_{1}^{* *}\left(e_{i i} \beta\left(e_{i i+1}\right) e_{i+1 i+1}\right)=e_{i i+1} .
$$

Since $m=\pi_{2} \mu_{1}$ from (23), we obtain

$$
m^{* *}\left(e_{i i} \beta\left(e_{i i+1}\right) e_{i+1 i+1}\right)=e_{i i+1} .
$$

Leibniz's rule, applied to the equation $e_{1 n}=e_{12} e_{23} \ldots e_{n-1 n}$, gives

$$
e_{11} \beta\left(e_{1 n}\right) e_{n n}=\sum_{i=1}^{n-1} e_{1 i} \beta\left(e_{i i+1}\right) e_{i+1 n}
$$

and so

$$
\begin{aligned}
m^{* *}\left(e_{11} \beta\left(e_{1 n}\right) e_{n n}\right) & =\sum_{i=1}^{n-1} e_{1 i} m^{* *}\left(\beta\left(e_{i i+1}\right)\right) e_{i+1 n} \\
& =(n-1) e_{1 n}
\end{aligned}
$$

from (39). Thus $\|\beta\| \geq n-1$ since $m$ is a contraction. From these inequalities it follows that

$$
n-1 \leq\|\beta\| \leq\|\gamma\| \leq\|\varepsilon\| \leq\|\delta\|+2,
$$

and so $\|\delta\| \geq n-3$, as required.

Remark 4.5. This result, combined with [5], shows that $A$ (arising from an infinite nest) is not 2-amenable and so there is a dual $A$-bimodule $M$ for which $H^{2}(A, M) \neq$ 0 . It seems very difficult to obtain such a module directly, although a posteriori $H^{2}\left(A, \operatorname{ker} \pi_{3}^{* *}\right) \neq 0$ from [5]. In the next section we study the simplest infinite nest algebra $T^{\infty}$ for which such modules can be constructed explicitly.

\section{Cohomology for the Weak operator Closed ideals of $T^{\infty}$}

In this section we calculate the cohomology groups $H^{n}\left(T^{\infty}, J\right)$ for many weak operator closed two-sided ideals $J$ of $T^{\infty}$, the nest algebra on an infinite dimensional separable Hilbert space, which naturally generalizes the $T_{m}$ 's. Consonant with what happens for the $T_{m}$ 's, $H^{2}\left(T^{\infty}, J\right)=0$ for such ideals. It seems likely that these results should extend to more general nest algebras. Indeed, the case of first cohomology groups is given in the more general context by Erdos and Power ([7], 
$\S 3)$, and they even allow the case where $J$ is replaced by a weak operator closed submodule of $B(H)$. However, to avoid technical complications, we will restrict to the case of $T^{\infty}$ and a weak operator closed ideal $J$ of $T^{\infty}$. For an excellent account of the theory of nest algebras, the reader is referred to the book [4] by K. Davidson.

We now recall the definition of $T^{\infty}$. Let $\left\{u_{i}\right\}$ be an orthonormal basis for an infinite dimensional, separable Hilbert space $H$. Let $H_{i}=\operatorname{Span}\left\{u_{1}, \ldots, u_{i}\right\}$ and $p_{i}: H \rightarrow H_{i}$ be the orthogonal projection onto $H_{i}$. Then

$$
T^{\infty}=\left\{A \in B(H): A\left(H_{i}\right) \subseteq H_{i} \text { for all } i \geq 1\right\} .
$$

Each $A \in T^{\infty}$ is representable as an infinite upper triangular matrix $\left\{A_{i j}\right\}$, where $A u_{i}=\sum_{k=1}^{i} A_{k i} u_{k}$. Let $F^{\infty}$ be the span of the matrix units $e_{i j}(1 \leq i \leq j)$ in $T^{\infty}$. Then for any $A \in T^{\infty}$ we have $p_{n} A p_{n} \in F^{\infty}$, and $p_{n} A p_{n} \rightarrow A$ in the strong operator topology.

It is easily checked that for $A \in T^{\infty}$,

$$
e_{i j} A e_{k \ell}=A_{j k} e_{i \ell}
$$

We wish to discuss the ideals $J$ in terms of certain sequences.

Let $I$ be the set of sequences $\alpha=\left\{\left(m_{1}, n_{1}\right),\left(m_{2}, n_{2}\right), \ldots\right\}$ (of finite or infinite length) such that:

(a) $m_{i} \in \mathbb{Z}^{+}, n_{i} \in \mathbb{Z}^{+} \cup\{\infty\}, m_{i} \leq n_{i}$ for all $i$;

(b) $m_{i}<m_{i+1}, n_{i}<n_{i+1}$ for all $i$.

Note that if $\infty$ occurs as a component of some $\left(m_{i}, n_{i}\right)$, then $\alpha$ is a finite sequence $\left\{\left(m_{1}, n_{1}\right), \ldots,\left(m_{k}, n_{k}\right)\right\}$ with $n_{k}=\infty$ and all the other $m_{i}, n_{i}$ finite. Note also that it is possible for $m_{i}<m_{i+1}<n_{i}$ to hold for some $i$. The members $\alpha$ of $I$ will be called ideal sequences.

Given $\alpha \in I$ as above, we define

$$
J_{\alpha}=\left\{A \in T^{\infty}: A_{i j}=0 \text { if } m_{k} \leq i \leq j \leq n_{k} \text { for some } k\right\} .
$$

We will show below that all weak* closed ideals $J$ in $T^{\infty}$ are of the form $J_{\alpha}$ for some unique $\alpha \in I$. Here are two examples. Suppose that $\alpha$ is the infinite sequence $\{(1,1),(2,2), \ldots\}$. Then $J_{\alpha}$ is the ideal of strictly upper triangular operators in $T^{\infty}$. If $\alpha=\{(1,3),(2,4),(5, \infty)\}$, then $J_{\alpha}$ is the set of all $A \in T^{\infty}$ such $A_{i j}=0$ whenever $1 \leq i \leq j \leq 3,2 \leq i \leq j \leq 4$ and $5 \leq i \leq j$.

Proposition 5.1. Every weak operator closed ideal of $T^{\infty}$ is of the form $J_{\alpha}$ for some unique $\alpha \in I$. Conversely, every $J_{\alpha}$ is a weak operator closed ideal of $T^{\infty}$.

Proof. Let $J$ be a weak operator closed ideal of $T^{\infty}$. By [7], Theorem 1.5 or [4], p. 193, there exists a left continuous, order homomorphism $\sigma: \mathcal{N} \rightarrow \mathcal{N}$ with $\sigma(0)=0$, where $\mathcal{N}$ is the nest of $T^{\infty}$. (The first two conditions on $\sigma$ mean that for any $\mathcal{K} \subseteq \mathcal{N}, \sup _{N \in \mathcal{K}} \sigma(N)=\sigma\left(\sup _{N \in \mathcal{K}} N\right)$ and $\sigma(E) \leq \sigma(F)$ if $E \leq F$ in $\mathcal{N}$.)

We can identify $\mathcal{N}$ with $\{0\} \cup \mathbb{Z}^{+} \cup\{\infty\}$. Then $\sigma(0)=0, \sigma$ is increasing and $\sigma(i) \rightarrow \sigma(\infty)$ as $i \rightarrow \infty$. Also

$$
J=\left\{A \in T^{\infty}: A\left(H_{i}\right) \subseteq H_{\sigma(i)} \text { for all } i\right\},
$$

where $H_{i}=p_{i}(H)$.

Let $P=\{i: \sigma(i)<i<\infty\}$ and $Z=\{\sigma(i): i \in P\}$. Order the elements of $P$ in an increasing sequence $\left\{z_{k}\right\}$. Let $A_{k}=\left\{i \in P: \sigma(i)=z_{k}\right\}$ and $m_{k}=\min A_{k}$. Since $\sigma$ is an order homomorphism, $A_{k}$ is an interval. Let $B_{k}=\left\{i \geq m_{k}: A\left(H_{i}\right) \subseteq H_{z_{k}}\right.$ 
for all $A \in J\}$. Clearly $B_{k}$ is also an interval $\left[m_{k}, n_{k}\right]$. Let $\alpha=\left\{\left(m_{k}, n_{k}\right)\right\}$. We claim that $\alpha$ is an ideal sequence.

By definition, $m_{k} \leq n_{k}$ for all $k$. Also, since $\sigma\left(m_{k}\right)<\sigma\left(m_{k+1}\right)$, we have $m_{k}<$ $m_{k+1}$. Next, if $n_{k}<m_{k+1}$, we have $n_{k}<n_{k+1}$. If $n_{k} \geq m_{k+1}$, then $A\left(H_{n_{k}}\right) \subseteq$ $H_{z_{k}} \subseteq H_{z_{k+1}}$, and so $n_{k} \leq n_{k+1}$. If $n_{k}=n_{k+1}$, then $A\left(H_{m_{k+1}}\right) \subseteq A\left(H_{n_{k+1}}\right) \subseteq H_{z_{k}}$. To derive a contradiction to $n_{k}=n_{k+1}$ we need only produce $A \in J$ with $u_{z_{k+1}} \in$ $A\left(H_{m_{k+1}}\right)$; take $A=e_{z_{k+1}, m_{k+1}}$. Thus $n_{k}<n_{k+1}$, and $\alpha$ is an ideal sequence.

We now claim that $J=J_{\alpha}$. Let $A \in J$. Suppose that for some $k$ we have $m_{k} \leq i \leq j \leq n_{k}$ ( $j$ finite). Then $A\left(H_{j}\right) \subseteq H_{z_{k}}$. Since $z_{k}<\sigma\left(m_{k}\right) \leq i$, we have $A_{i j}=0$, and so $A \in J_{\alpha}$ and $J \subseteq J_{\alpha}$. Conversely, let $A \in J_{\alpha}$. To show that $A \in J$, we only need prove that $A\left(H_{i}\right) \subseteq H_{\sigma(i)}$ for $i \in P$. Suppose that $i \in P$ and $\sigma(i)=z_{k}$. Then $m_{k} \leq i$, while, since for $A^{\prime} \in J, A^{\prime}\left(H_{i}\right) \subseteq H_{\sigma(i)}=H_{z_{k}}$ and $i \in P$, we have $i \in A_{k}^{\prime}$ so that $i \leq n_{k}$. So $m_{k} \leq i \leq n_{k}$. For $m_{r} \leq j \leq i \leq n_{k}$ we have

$$
A u_{j}=\sum_{k=1}^{j} A_{k j} u_{k}=\sum_{k=1}^{m_{k}} A_{k j} u_{k} \in A\left(H_{m_{k}}\right) \subseteq H_{z_{k}}
$$

since $A_{k j}=0$ if $m_{k} \leq k \leq j$. So $A\left(H_{i}\right) \subseteq A\left(H_{m_{k}}\right) \subseteq H_{\sigma(i)}$. So $A \in J$.

Let $\alpha$ be an ideal sequence $\left\{\left(m_{r}, n_{r}\right)\right\}$ and let

$$
T_{m_{r}, n_{r}}=\operatorname{Span}\left\{e_{i j}: m_{r} \leq i \leq j \leq n_{r}\right\} .
$$

Let $U=\{(i, j): 1 \leq i \leq j\}, V=\left\{(i, j)\right.$ : for some $\left.r, m_{r} \leq i \leq j \leq n_{r}\right\}$ and $W=U \backslash V$. For any subset $X$ of $U$, let

$$
Z_{X}=\left\{A \in T^{\infty}: A_{i j}=0 \text { for all }(i, j) \in X\right\} .
$$

Clearly $Z_{X}$ is a weak operator closed subspace of $T^{\infty}$. By definition, $J_{\alpha}=Z_{V}$.

Let $R_{\alpha}=Z_{W}$. In order to use the long exact sequence property of cohomology, we will require $T^{\infty}$ to be the Banach space direct sum $J_{\alpha} \oplus R_{\alpha}$ in a canonical way. (This is a significant requirement; K.R. Davidson has pointed out to the authors that weak operator closed ideals in nest algebras are not in general complemented in the Banach space category.)

For each $r$, let $e_{r}=\sum_{i=m_{r}}^{n_{r}} e_{i i}$. (If $n_{r}=\infty$ then the convergence of this sum is taken in the strong operator topology.) Define, for each $N, P_{N}: T^{\infty} \rightarrow T^{\infty}$ by:

$$
P_{N}(A)=\sum_{r=1}^{N} e_{r} A e_{r}-\sum_{r=1}^{N-1} g_{r} A g_{r}
$$

where $g_{r}=e_{r} e_{r+1}$. Since $e_{r} A e_{r} \in T_{m_{r}, n_{r}}$ for all $A$, it is clear that $P_{N} \in B\left(T^{\infty}, R_{\alpha}\right)$. The map $P_{N}$ takes $A \in T^{\infty}$ into the operator whose $(i, j)^{\text {th }}$ component is $A_{i j}$ if $m_{r} \leq i \leq j \leq n_{r}$ for some $1 \leq r \leq N$ and is 0 otherwise. (The second sum in (42) removes "overlaps" between consecutive $T_{m_{r}, n_{r}}$ 's.)

Proposition 5.2. Suppose that $\sup \left\{\left\|P_{N}\right\|: N \geq 1\right\}<\infty$. Then the map $P$, where, for $A \in T^{\infty}, P(A)_{i j}=A_{i j}$ for $(i, j) \in V$ and is zero otherwise, is a projection in $B\left(T^{\infty}\right)$ whose range is $R_{\alpha}$ and whose kernel is $J_{\alpha}$.

Proof. If $\alpha$ is a finite sequence, then $P=P_{N}$ for some $N$ and the result is obvious.

Suppose, then, that $\alpha=\left\{\left(m_{r}, n_{r}\right)\right\}$ is an infinite sequence (so that, in particular, $n_{r}<\infty$ for all $r$ and $\left.m_{r}, n_{r} \rightarrow \infty\right)$. Using the uniform boundedness of the $P_{N}$ 's 
and Tychonoff's Theorem, there exists $P \in B\left(T^{\infty}\right)$ and a subnet $\left\{P_{\alpha(\delta)}\right\}$ of $\left\{P_{N}\right\}$

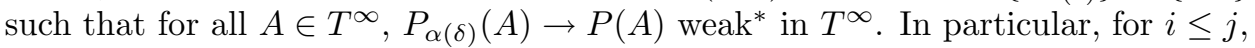

$$
P(A)_{i j}=\left\langle P(A) u_{j}, u_{i}\right\rangle=\lim _{\delta}\left\langle P_{\alpha(\delta)} u_{j}, u_{i}\right\rangle=\lim \left(P_{\alpha(\delta)}(A)\right)_{i j} .
$$

For large enough $\delta, P_{\alpha(\delta)}(A)_{i j}=A_{i j}$ if $(i, j) \in V$, and is 0 otherwise. Thus $P(A) \in R_{\alpha}$ and $P: T^{\infty} \rightarrow R_{\alpha}$. Obviously, $P$ is a projection on $B\left(T^{\infty}\right)$ onto $R_{\alpha}$. Further, ker $P$ is precisely the set of those $A$ 's for which $A_{i j}=0((i, j) \in V)$, and so is $J_{\alpha}$.

If $\sup \left\{\left\|P_{N}\right\|: \quad N \geq 1\right\}<\infty$, then we will say that the sequence $\alpha$ is bounded. The next result provides many examples of bounded $\alpha$ 's. Let

$$
M_{\alpha}=\sup _{r \geq 1}\left|\left\{s: m_{r}<m_{s} \leq n_{r}\right\}\right| .
$$

Then $M_{\alpha} \in\{0\} \cup \mathbb{Z}^{+} \cup\{\infty\}$.

Proposition 5.3. Suppose that $M_{\alpha}<\infty$. Then $\alpha$ is bounded.

Proof. Clearly $M_{\alpha}$ is a "max" and an integer $\geq 0$. Let $p=M_{\alpha}+1$. By (42), it is sufficient to show that $\left\|\sum_{r=1}^{N} e_{r} A e_{r}\right\|,\left\|\sum_{r=1}^{N-1} e_{r} e_{r+1} A e_{r} e_{r+1}\right\|$ are at most $K\|A\|$, where $K$ is independent of $N$. Now

$$
\left\|\sum_{r=1}^{N} e_{r} A e_{r}\right\| \leq \sum_{a=1}^{p}\left\|\sum_{k=0}^{N}\left(e_{a+k p} A e_{a+k p}\right)^{\prime}\right\|,
$$

where $\left(e_{a+k p} A e_{a+k p}\right)^{\prime}=e_{a+k p} A e_{a+k p}$ if $a+k p \leq N$ and is 0 otherwise. Now if $k<\ell$, then $e_{a+k p} e_{a+\ell p}=0$, since otherwise there would be an $i$ such that

$$
m_{a+k p} \leq i \leq n_{a+k p}, \quad m_{a+\ell p} \leq i \leq n_{a+\ell p},
$$

and so

$$
m_{a+k p}<m_{a+\ell p} \leq i \leq n_{a+k p},
$$

and

$$
\left|\left\{s: m_{a+k p}<m_{s} \leq n_{a+k p}\right\}\right| \geq p>M,
$$

giving a contradiction.

Hence each $\sum_{k=1}^{N}\left(e_{a+k p} A e_{a+k p}\right)^{\prime}$ is a direct sum of the operators $\left(e_{a+k p} A e_{a+k p}\right)^{\prime}$, and (44) gives

$$
\left\|\sum_{r=1}^{N} e_{r} A e_{r}\right\| \leq p\|A\| .
$$

Similarly, $\left\|\sum_{r=1}^{N-1} e_{r} e_{r+1} A e_{r} e_{r+1}\right\| \leq p\|A\|$, and $\alpha$ is bounded.

The case where $M_{\alpha}=0$ is equivalent to the requirement that $n_{r}<m_{r+1}$ for all $r$. A simple example of this is the sequence $\alpha=\{(1,1),(2,2),(3,3), \ldots\}$ giving the ideal of strictly upper triangular operators in $T^{\infty}$.

Suppose that $\alpha$ is bounded and let $J=J_{\alpha}$. Since $T^{\infty}=R_{\alpha} \oplus J_{\alpha}$, the quotient module $T^{\infty} / J_{\alpha}$ can be identified as a Banach space with $R_{\alpha}$ in the canonical way. As a Banach $T^{\infty}$-module, the module action on $R_{\alpha}$ is just that of ordinary matrix 
multiplication with $(i, j)$-entries equated to 0 if $(i, j) \notin V$. More precisely, if $A \in$ $R_{\alpha}, B \in T^{\infty}$ and the module action is denoted by $\cdot$, then $A \cdot B=P(A B), B \cdot A=$ $P(B A)$.

Lemma 5.4. Let $\alpha$ be bounded. Then for $n \geq 2$,

$$
H^{n}\left(T^{\infty}, J_{\alpha}\right)=H^{n-1}\left(T^{\infty}, R_{\alpha}\right) .
$$

Proof. The sequence $0 \rightarrow J_{\alpha} \rightarrow T^{\infty} \rightarrow R_{\alpha} \rightarrow 0$ is admissible (in the sense that it splits in the Banach space category). The long exact sequence for Ext in the second variable ([12], p. 156, [13], p. 12) then gives the exact sequence

$$
\cdots \rightarrow H^{n-1}\left(T^{\infty}, T^{\infty}\right) \rightarrow H^{n-1}\left(T^{\infty}, R_{\alpha}\right) \rightarrow H^{n}\left(T^{\infty}, J_{\alpha}\right) \rightarrow H^{n}\left(T^{\infty}, T^{\infty}\right) \rightarrow \cdots
$$

A result of Lance and Nielsen ([15], [16]; see [1] for earlier work) implies that $H^{r}\left(T^{\infty}, T^{\infty}\right)=0$ for all $r \geq 1$. (We note that this result has been extended to certain classes of CSL algebras by Gilfeather, Hopenwasser and Larson [8].) Hence by $(46), H^{n-1}\left(T^{\infty}, R_{\alpha}\right)=H^{n}\left(T^{\infty}, J_{\alpha}\right)$.

The preceding lemma (for $\alpha$ bounded) reduces the study of $T^{\infty}$-cohomology for $J_{\alpha}$ to that of cohomology for $R_{\alpha}$. The advantages of this are not just that the level of the cohomology groups is reduced by 1 . The module $R_{\alpha}$ is built up out of the upper triangular algebras $T_{m_{r}, n_{r}}$ which are subalgebras of $T^{\infty}$, and the LanceNielsen result used above gives precise information about the cohomology groups of such algebras. The objective then is to compute $R_{\alpha}$-cohomology in terms of sequences of cocycles of the $T_{m_{r}, n_{r}}$ 's which "mesh together" to give a $T^{\infty}$-cocycle into $R_{\alpha}$. (These sequences are called $\alpha$-coherent.) For this to work, we need to restrict to multimodular cocycles. A cocycle will be called multimodular if it is $B$ multimodular, where $B$ is the core (diagonal subalgebra) of the triangular algebra.

Lemma 5.5. There exists a net of elements of the form $\left(p_{r_{1}}, \ldots, p_{r_{n+1}}\right)$ such that for all multimodular $f \in C^{n}\left(T^{\infty}, R_{\alpha}\right)$, the net of operators

$$
f\left(p_{r_{1}} a_{1} p_{r_{2}}, p_{r_{2}} a_{2} p_{r_{3}}, \ldots, p_{r_{n}} a_{n} p_{r_{n+1}}\right)
$$

converges to $f\left(a_{1}, \ldots, a_{n}\right)$ in the strong operator topology for all $a_{1}, \ldots, a_{n} \in T^{\infty}$.

Proof. Let $a_{1}, \ldots, a_{n} \in T^{\infty}, \xi_{1}, \ldots, \xi_{k} \in H$ and $\varepsilon>0$. Let $f \in C^{n}\left(T^{\infty}, R_{\alpha}\right)$ be multimodular. Choose $r_{n+1}$ large enough so that

$$
\left\|f\left(a_{1}, \ldots, a_{n}\right) p_{r_{n+1}} \xi_{i}-f\left(a_{1}, \ldots, a_{n}\right) \xi_{i}\right\|<\frac{\varepsilon}{2^{n}}
$$

for $1 \leq i \leq k$. Now $a_{n} p_{r_{n+1}}$ is compact, and so $p_{s}\left(a_{n} p_{r_{n+1}}\right) \rightarrow a_{n} p_{r_{n+1}}$ in norm as $s \rightarrow \infty$. By the norm continuity of $f$, there exists $r_{n}$ so that

$$
\left\|f\left(a_{1}, \ldots, a_{n-1}, p_{r_{n}} a_{n} p_{r_{n+1}}\right) \xi_{i}-f\left(a_{1}, \ldots, a_{n-1}, a_{n} p_{r_{n+1}}\right) \xi_{i}\right\|<\frac{\varepsilon}{2^{n-1}}
$$

for $1 \leq i \leq k$. Since $f$ is multimodular and $p_{r_{n}}^{2}=p_{r_{n}}$, (48) gives

$$
\left\|f\left(a_{1}, \ldots, a_{n-1} p_{r_{n}}, p_{r_{n}} a_{n} p_{r_{n+1}}\right) \xi_{i}-f\left(a_{1}, \ldots, a_{n-1}, a_{n} p_{r_{n+1}}\right) \xi_{i}\right\|<\frac{\varepsilon}{2^{n-1}}
$$

for $1 \leq i \leq k$. Repeating the same argument recursively, we construct $r_{k}$ 's so that for each $j$,

$$
\begin{aligned}
& \| f\left(a_{1}, \ldots, a_{j-1}, a_{j} p_{r_{j+1}}, p_{r_{j+1}} a_{j+1} p_{r_{j+2}}, \ldots, p_{r_{n}} a_{n} p_{r_{n+1}}\right) \xi_{i} \\
& \quad-f\left(a_{1}, \ldots, a_{j}, a_{j+1} p_{r_{j+2}}, p_{r_{j+2}} a_{j+2} p_{r_{j+3}}, \ldots, p_{r_{n}} a_{n} p_{r_{n+1}}\right) \xi_{i} \|<\frac{\varepsilon}{2^{j}} .
\end{aligned}
$$


Summing up the inequalities (50) and using the triangular inequality gives

$$
\left\|f\left(a_{1}, \ldots, a_{n}\right) \xi_{i}-f\left(p_{r_{1}} a_{1} p_{r_{2}}, \ldots, p_{r_{n}} a_{n} p_{r_{n+1}}\right) \xi_{i}\right\|<\varepsilon
$$

for $1 \leq i \leq k$.

It is easy to check that the choice of the $r_{k}$ 's can be made simultaneously for any finite number of $f$ 's and for any finite number of sequences $\left(a_{1}, \ldots, a_{n}\right)$. The existence of the desired net now follows.

Definition. Let $\alpha$ be an ideal sequence. A sequence $\left\{f_{r}\right\}$ is called an $\alpha$-coherent sequence of $n$-cochains if:

(a) $f_{r} \in C^{n}\left(T_{m_{r}, n_{r}}, T_{m_{r}, n_{r}}\right)$;

(b) for all $r, f_{r}\left(a_{1}, \ldots, a_{n}\right)=f_{r+1}\left(a_{1}, \ldots, a_{n}\right)$ whenever all of the $a_{i} \in T_{m_{r}, n_{r}} \cap$ $T_{m_{r+1}, n_{r+1}}, 1 \leq i \leq n$

(c) $\sup _{r \geq 1}\left\|f_{r}\right\|=K<\infty$; $r \geq 1$

(d) each $f_{r}$ is multimodular.

The sequence $\left\{f_{r}\right\}$ is called an $\alpha$-coherent sequence of $n$-cocycles if every $f_{r}$ is a cocycle.

In the next result we will use the following notation. For an $\alpha$-coherent sequence of $n$-cochains $\left\{f_{n}\right\}$ we define $f \in C^{n}\left(T^{\infty}, R_{\alpha}\right)$ by the strong operator convergent sum

$$
f\left(a_{1}, \ldots, a_{n}\right)=\sum_{r \geq 1}\left[f_{r}\left(e_{r} a_{1} e_{r}, \ldots, e_{r} a_{n} e_{r}\right)-f_{r+1}\left(g_{r} a_{1} g_{r}, \ldots, g_{r} a_{n} g_{r}\right)\right] .
$$

Given $f \in C^{n}\left(T^{\infty}, R_{\alpha}\right)$, we let $f_{r}$ denote the restriction of $f$ to $T_{m_{r}, n_{r}}$. Similar definitions apply with $f$ replaced by $h$ or $k$.

Proposition 5.6. Suppose that $M_{\alpha}<\infty$.

(i) If $\left\{f_{r}\right\}$ is an $\alpha$-coherent sequence of $n$-cochains, then $f \in C^{n}\left(T^{\infty}, R_{\alpha}\right)$ is multimodular and satisfies

$$
e_{r} f\left(a_{1}, \ldots, a_{n}\right) e_{r}=f\left(a_{1}, \ldots, a_{n}\right) e_{r}=f_{r}\left(e_{r} a_{1} e_{r}, \ldots, e_{r} a_{n} e_{r}\right) .
$$

(ii) If $f \in C^{n}\left(T^{\infty}, R_{\alpha}\right)$ is multimodular, then $\left\{f_{r}\right\}$ is a $\alpha$-coherent and (51) holds.

(iii) If $\left\{h_{r}\right\}$ and $\left\{k_{r}\right\}$ are $\alpha$-coherent sequences of $(n-1)$-cochains and $n$-cochains respectively which satisfy $\delta h_{r}=k_{r}$, then $\delta h=\delta k$.

(iv) If $h \in C^{n-1}\left(T^{\infty}, R_{\alpha}\right)$ and $k \in C^{n}\left(T^{\infty}, R_{\alpha}\right)$ are multimodular and satisfy $\delta h=k$, then $\delta h_{r}=k_{r}$ for all $r$.

Proof. Suppose firstly that $\left\{f_{r}\right\}$ is an $\alpha$-coherent sequence of $n$-cochains. Note that in (51), $e_{r} a_{i} e_{r} \in T_{m_{r}, n_{r}}, g_{r} a_{i} g_{r} \in T_{m_{r+1}, n_{r+1}} \cap T_{m_{r}, n_{r}}$, so that the summands make sense. Using the multimodular property of each $f_{r}$, the sum in (51) is of the form $\sum_{r \geq 1}\left(e_{r} A_{r} e_{r}-g_{r} B_{r} g_{r}\right)$ with $\left\|A_{r}\right\|,\left\|B_{r}\right\| \leq K\left\|a_{1}\right\| \ldots\left\|a_{n}\right\|$. Using the argument of Proposition 5.2, we have

$$
\left\|\sum_{r=1}^{N}\left(e_{r} A_{r} e_{r}-g_{r} B_{r} g_{r}\right)\right\| \leq 2\left(M_{\alpha}+1\right) K\left\|a_{1}\right\| \ldots\left\|a_{n}\right\|
$$

and the series in (51) converges in the strong operator topology.

Clearly, $f \in C^{n}\left(T^{\infty}, R_{\alpha}\right)$. We now claim that $f$ is multimodular. Indeed, let $b \in B$. Then $b$ and each $e_{r}$ commute, and $b e_{r} \in B_{r}$. Then for each $r$,

$$
f_{r}\left(e_{r} b a_{1} e_{r}, \ldots, e_{r} a_{n} e_{r}\right)=b e_{r} f_{r}\left(e_{r} a_{1} e_{r}, \ldots, e_{r} a_{n} e_{r}\right)+b f_{r}\left(e_{r} a_{1} e_{r}, \ldots, e_{r} a_{n} e_{r}\right),
$$


and it follows from (51) that

$$
b f\left(a_{1}, \ldots, a_{n}\right)=f\left(b a_{1}, \ldots, a_{n}\right) .
$$

The other multimodular properties are proved similarly.

Next we claim that

$$
e_{r} f\left(a_{1}, \ldots, a_{n}\right) e_{r}=f_{r}\left(e_{r} a_{1} e_{r}, \ldots, e_{r} a_{n} e_{r}\right) .
$$

Firstly, let $a \in T^{\infty}$ and $k, r$ be given. Suppose that $r \geq k$. Then $e_{r} e_{k}=\sum_{m_{r}}^{n_{k}} e_{p p}$, and if $\left(e_{r} e_{k} a e_{k}\right)_{i j} \neq 0$, then $m_{r} \leq i \leq j \leq n_{k}$, so that since $a \in T^{\infty},\left(e_{r} e_{k} a e_{k}\right)_{i j}=$ $\left(e_{r} e_{k} a e_{r} e_{k}\right)_{i j}$. It follows that if $r \geq k$, then $e_{r} e_{k} a e_{k}=e_{r} e_{k} a e_{r} e_{k}$. Similarly, if $r \leq k$ then $e_{k} a e_{r} e_{k}=e_{r} e_{k} a e_{r} e_{k}$. It follows by pushing $e_{r} e_{k}$ through from left to right or right to left (using $\alpha$-coherence) that

$$
\begin{aligned}
& e_{r} f_{k}\left(e_{k} a_{1} e_{k}, \ldots, e_{k} a_{n} e_{k}\right) e_{r}=f_{k}\left(e_{r} e_{k} a_{1} e_{r} e_{k}, \ldots, e_{r} e_{k} a_{n} e_{r} e_{k}\right) \\
& \quad=f_{r}\left(e_{r} e_{k} a_{1} e_{r} e_{k}, \ldots, e_{r} e_{k} a_{n} e_{r} e_{k}\right)=e_{k} f_{r}\left(e_{r} a_{1} e_{r}, \ldots, e_{r} a_{n} e_{r}\right) e_{k} .
\end{aligned}
$$

It also follows that

$$
\begin{aligned}
& e_{r} f_{k+1}\left(g_{k} a_{1} g_{k}, \ldots, g_{k} a_{n} g_{k}\right) e_{r}=e_{k} f_{r}\left(e_{r} e_{k+1} a_{1} e_{r} e_{k+1}, \ldots, e_{r} e_{k+1} a_{n} e_{r} e_{k+1}\right) e_{k} \\
& \quad=g_{k} e_{r} f_{r}\left(e_{r} a_{1} e_{r}, \ldots, e_{r} a_{n} e_{r}\right) g_{k} e_{r} .
\end{aligned}
$$

Let $S=\left\{k: T_{m_{k}, n_{k}} \cap T_{m_{r}, n_{r}} \neq \emptyset\right\}$. Since the $m_{k}$ 's are strictly increasing, $S$ is finite. Let $p_{1}=\min S, p_{2}=\max S$. We claim that $S=\left\{k: p_{1} \leq k \leq p_{2}\right\}$. Indeed, suppose that $k \in S$. Clearly, $r \in S$, and $m_{p_{1}} \leq m_{r}, n_{p_{1}} \leq n_{r}$. Since $p_{1}=\min S$, we must have $m_{r} \leq n_{p_{1}}$. Similarly, since $p_{2}=\max S, m_{r} \leq m_{p_{2}}, n_{r} \leq n_{p_{2}}$ and we must have $m_{p_{2}} \leq n_{r}$. Thus $m_{r} \leq n_{p_{1}} \leq n_{k}, m_{k} \leq m_{p_{2}} \leq n_{r}$ and the integer intervals $\left[m_{k}, m_{k}\right],\left[m_{r}, n_{r}\right]$ overlap. Thus $S=\left[p_{1}, p_{2}\right]$.

From the above calculation and strong operator continuity for multiplication,

$$
\begin{aligned}
& e_{r} f\left(a_{1}, \ldots, a_{n}\right) e_{r} \\
& \quad=\sum_{k=p_{1}}^{p_{2}}\left[e_{k} f_{r}\left(e_{r} a_{1} e_{r}, \ldots, e_{r} a_{n} e_{r}\right) e_{k}-g_{k} f_{r}\left(e_{r} a_{1} e_{r}, \ldots, e_{r} a_{n} e_{r}\right) g_{k}\right] .
\end{aligned}
$$

Now let $m_{r} \leq i \leq j \leq n_{r}$ and $S_{i j}=\left\{k: m_{k} \leq i \leq j \leq n_{k}\right\}$. It is obvious that $S_{i j}$ is an integer interval $\left[p_{i j}, q_{i j}\right] \subseteq\left[p_{1}, p_{2}\right] .\left(S_{i j} \neq \emptyset\right.$ since it contains $r$. $)$ Then

$$
\begin{aligned}
\left(e_{r} f\left(a_{1}, \ldots, a_{n}\right) e_{r}\right)_{i j}= & \sum_{k=p_{i j}}^{q_{i j}-1}\left[f_{r}\left(e_{r} a_{1} e_{r}, \ldots, e_{r} a_{n} e_{r}\right)_{i j}-f_{r}\left(e_{r} a_{1} e_{r}, \ldots, e_{r} a_{n} e_{r}\right)_{i j}\right] \\
& +\left(f_{r}\left(e_{r} a_{1} e_{r} \ldots, e_{r} a_{n} e_{r}\right)_{i j}-0\right) \\
= & f_{r}\left(e_{r} a_{1} e_{r}, \ldots, e_{r} a_{n} e_{r}\right) .
\end{aligned}
$$

Since $\left(e_{r} f\left(a_{1}, \ldots, a_{n}\right) e_{r}\right)_{i j} \neq 0$ implies $e_{i j} \in T_{m_{r}, n_{r}}$, it follows that

$$
e_{r} f\left(a_{1}, \ldots, a_{n}\right) e_{r}=f_{r}\left(e_{r} a_{1} e_{r}, \ldots, e_{r} a_{n} e_{r}\right) \text {. }
$$

This proves (i).

Conversely, let $f \in C^{n}\left(T^{\infty}, R_{\alpha}\right)$ be multimodular. Let $f_{r}=f_{\mid T_{m_{r}, n_{r}} \times \cdots \times T_{m_{r}, n_{r}}}$. Since, for $a_{i} \in T_{m_{r}, n_{r}}$,

$$
f\left(a_{1}, \ldots, a_{n}\right)=f\left(e_{r} a_{1}, \ldots, a_{n} e_{r}\right)=e_{r} f\left(a_{1}, \ldots, a_{n}\right) e_{r},
$$


it follows that $f_{r} \in C^{n}\left(T_{m_{r}, n_{r}}, T_{m_{r}, n_{r}}\right)$. It is easy to check that the sequence $\left\{f_{r}\right\}$ is an $\alpha$-coherent sequence of $n$-cochains. Define (using the above) $g \in C^{n}\left(T^{\infty}, R_{\alpha}\right)$ by:

$$
g\left(a_{1}, \ldots, a_{n}\right)=\sum_{r \geq 1}\left[f_{r}\left(e_{r} a_{1} e_{r}, \ldots, e_{r} a_{n} e_{r}\right)-f_{r+1}\left(g_{r} a_{1} g_{r}, \ldots, g_{r} a_{n} g_{r}\right)\right] .
$$

The cochain $g$ is multimodular. We now show that $f=g$. To this end, it is sufficient to show that for any $r_{k}$ 's,

$$
f\left(p_{r_{1}} a_{1} p_{r_{2}}, \ldots, p_{r_{n}} a_{n} p_{r_{n+1}}\right)=g\left(p_{r_{1}} a_{1} p_{r_{2}}, \ldots, p_{r_{n}} a_{n} p_{r_{n+1}}\right) .
$$

Clearly, (52) is equivalent to

$$
(f-g)\left(e_{i_{1} i_{1}} a_{1} e_{j_{1} j_{1}}, e_{i_{2} i_{2}} a_{2} e_{j_{2} j_{2}}, \ldots, e_{i_{n} i_{n}} a_{n} e_{j_{n} j_{n}}\right)=0
$$

for all possible $i_{k}, j_{k}$. Suppose that the left-hand side of (53) is non-zero. Then since $(f-g)$ is multimodular, $j_{k}=i_{k+1}$. Since the $a_{i}$ 's are upper triangular and $(f-g)$ is multimodular, we can suppose that $i_{k}<i_{k+1}$ for all $k$. We thus have to show that in $R_{\alpha}$,

$$
(f-g)\left(e_{i_{1} i_{1}} a_{1} e_{i_{2} i_{2}}, e_{i_{2} i_{2}} a_{2} e_{i_{3} i_{3}}, \ldots, e_{i_{n} i_{n}} a_{n} e_{i_{n+1} i_{n+1}}\right)=0 .
$$

Suppose that $e_{i_{1} i_{n+1}} \notin R_{\alpha}$. Then, identifying $R_{\alpha}$ with $T^{\infty} / J_{\alpha}$,

$$
\begin{aligned}
& (f-g)\left(e_{i_{1} i_{1}} a_{1} e_{i_{2} i_{2}}, \ldots, e_{i_{n} i_{n}} a_{n} e_{i_{n+1} i_{n+1}}\right) \\
& \quad=e_{i_{1} i_{1}}(f-g)\left(e_{i_{1} i_{1}} a_{1} e_{i_{2} i_{2}}, \ldots, e_{i_{n} i_{n}} a_{n} e_{i_{n+1} i_{n+1}}\right) e_{i_{n+1} i_{n+1}} \\
& \quad \in\left(\mathbb{C} e_{i_{1} i_{n+1}}+J_{\alpha}\right) \subseteq J_{\alpha}
\end{aligned}
$$

so that (54) follows.

Suppose then that $e_{i_{1} i_{n+1}} \in R_{\alpha}$. Then $e_{i_{1} i_{n+1}} \in T_{m_{r}, n_{r}}$ for some $r$. Now

$$
e_{i_{k} i_{k}} a_{k} e_{i_{k+1} i_{k+1}}=\left(a_{k}\right)_{i_{k} i_{k+1}} e_{i_{k} i_{k+1}}
$$

and since $m_{r} \leq i_{1}<i_{2}<\cdots<i_{n+1} \leq n_{r}$, all the $e_{i_{k} i_{k}} a_{k} e_{i_{k+1} i_{k+1}}$ 's belong to $T_{m_{r}, n_{r}}$, and (54) follows since both $f$ and $g$ coincide with $f_{r}$ on $T_{m_{r}, n_{r}}$. This proves (ii).

Next suppose that $\left\{h_{r}\right\},\left\{k_{r}\right\}$ are $\alpha$-coherent sequences of $(n-1)$-cochains and $n$-cochains respectively. Let $h, k$ be defined as in (51). Then for any $r$, using the 
earlier results of this proposition,

$$
\begin{aligned}
e_{r} \delta h( & \left.a_{1}, \ldots, a_{n}\right) e_{r} \\
= & e_{r}\left[a_{1} h\left(a_{2}, \ldots, a_{n}\right)-\sum_{i=1}^{n-1}(-1)^{i-1} h\left(a_{1}, \ldots, a_{i} a_{i+1}, \ldots, a_{n}\right)\right. \\
& \left.+(-1)^{n-1} h\left(a_{1}, \ldots, a_{n-1}\right) a_{n}\right] e_{r} \\
= & e_{r} a_{1} e_{r}\left(e_{r} h\left(a_{2}, \ldots, a_{n}\right) e_{r}\right)-\sum_{i=1}^{n-1}(-1)^{i-1} e_{r} h\left(a_{1}, \ldots, a_{i} a_{i+1}, \ldots, a_{n}\right) e_{r} \\
& +(-1)^{n-1} e_{r} h\left(a_{1}, \ldots, a_{n-1}\right) e_{r} a_{n} e_{r} \\
= & e_{r} a_{1} e_{r} h_{r}\left(e_{r} a_{2} e_{r}, \ldots, e_{r} a_{n} e_{r}\right) \\
& -\sum_{i=1}^{n} h_{r}\left(e_{r} a_{1} e_{r}, \ldots, e_{r} a_{i} a_{i+1} e_{r}, \ldots, e_{r} a_{n} e_{r}\right) \\
& +(-1)^{n-1} e_{r} h_{r}\left(e_{r} a_{1} e_{r}, \ldots, e_{r} a_{n-1} e_{r}\right) e_{r} a_{n} e_{r} \\
= & \delta h_{r}\left(e_{r} a_{1} e_{r}, \ldots, e_{r} a_{n} e_{r}\right)
\end{aligned}
$$

(since $e_{r} a_{i} a_{i+1} e_{r}=e_{r} a_{i} e_{r} a_{i+1} e_{r}$ ). It follows that $\delta h$ is associated with the $\alpha$ coherent sequence $\delta h_{r}$, and so $\delta h=k$ if and only if $\delta h_{r}=k_{r}$. This proves (iii) and (iv)

Theorem 5.7. Suppose $n_{r}<m_{r+1}$ for all but finitely many $r$. Then $H^{n}\left(T^{\infty}, R_{\alpha}\right)$ $=0$ for all $n \geq 1$.

Proof. Let $n \geq 1$ and $f \in Z^{n}\left(T^{\infty}, R_{\alpha}\right)$. We shall show that $f$ is a coboundary.

Now $f$ is given by (51), where $f_{r}=f_{\mid T_{m_{r}, n_{r}} \times \cdots \times T_{m_{r}, n_{r}}}$. By the Lance-Nielsen result above, there exists $g_{r} \in C^{n-1}\left(T_{m_{r}, n_{r}}, T_{m_{r}, n_{r}}\right)$ such that $f_{r}=\delta g_{r}$ and $\left\|g_{r}\right\| \leq$ $\left\|f_{r}\right\|$. (The latter inequality follows from the explicit formula for $g_{r}$ given in the proof of [15], Theorem 2.1.) By averaging appropriately over the unitary group of $T_{m_{r}, n_{r}}$ it follows ([9], Lemma 1.2$)$ that $g_{r}$ can be taken to be $B_{r}$-multimodular. We now show that the $g_{r}$ 's can be adjusted to give an $\alpha$-coherent family $\left\{g_{r}^{\prime}\right\}$. By Proposition 5.6, such a family $\left\{g_{r}^{\prime}\right\}$ defines a $g \in C^{n-1}\left(T^{\infty}, R_{\alpha}\right)$ with $\delta g=f$, so that $f$ is a coboundary.

We start with $g_{1}^{\prime}=g_{1}$. Let $S=T_{m_{1}, n_{1}} \cap T_{m_{2}, n_{2}}$. By the multimodular property, $g_{i}(S) \subseteq S(i=1,2)$. Write $\left(g_{1}-g_{2}\right)=g_{1_{\mid S}}-g_{2_{\mid S}}$. Since $f_{1_{\mid S}}=f_{2_{\mid S}}$, we have $\delta\left(g_{1}-g_{2}\right)=0$. We deal with the case $n \geq 2$, the case $n=1$ being an easy adaptation.

Now $S \cong T_{r}$ for some $r$, and by the Lance-Nielsen result, there exists $h \in$ $C^{n-2}(S, S)$ such that $\delta h=\left(g_{1}-g_{2}\right)$. Note that $S$ is finite-dimensional. (The existence of $\left(m_{2}, n_{2}\right)$ implies that $T_{m_{1}, n_{1}}$ is finite-dimensional.) So $C_{n-2}(S)$ is a finite-dimensional subspace of $C_{n-2}\left(T_{m_{2}, n_{2}}\right)$. We can identify $h$ as an element of $B\left(C_{n-2}(S), S\right)$. By the Hahn-Banach Theorem, there is $h^{\prime} \in B\left(C_{n-2}\left(T_{m_{2}, n_{2}}\right), S\right)=$ $C^{n-2}\left(T_{m_{2}, n_{2}}, S\right)$ such that $h_{\mid C_{n-2}(S)}^{\prime}=h$. Regard $h^{\prime} \in C^{n-2}\left(T_{m_{2}, n_{2}}, T_{m_{2}, n_{2}}\right)$. Now perform the averaging of $h^{\prime}$ as in [9], Lemma 1.2 to obtain a multimodular map 
$h^{\prime \prime} \in C^{n-2}\left(T_{m_{2}, n_{2}}, T_{m_{2}, n_{2}}\right)$ equivalent to $h^{\prime}$, where

$$
\begin{aligned}
h^{\prime \prime}\left(A_{1}, \ldots, A_{n-2}\right)=\int \ldots \int U_{1}^{*} h^{\prime}\left(U_{1} A_{1} U_{2}^{*}, U_{2} A_{2} U_{3}^{*},\right. \\
\left.\ldots, U_{n-2} A_{n-2} U_{n-1}^{*}\right) U_{n-1} d m\left(U_{1}\right) \ldots d m\left(U_{n-1}\right)
\end{aligned}
$$

with $m$ an invariant mean on the unitary group of $B_{2}$. Then $\delta h^{\prime \prime}=\delta h^{\prime}$, and since $h$ is multimodular, $h_{\mid C^{n-2}(S)}^{\prime \prime}=h$. Let $g_{2}^{\prime} \in C^{n-1}\left(T_{m_{2}, n_{2}}, T_{m_{2}, n_{2}}\right)$ be given by $g_{2}^{\prime}=g_{2}+\delta h^{\prime \prime}$. Then $g_{2}^{\prime}$ is multimodular, and if $a_{1}, \ldots, a_{n-1} \in S$, then

$$
\begin{aligned}
g_{2}^{\prime}\left(a_{1}, \ldots, a_{n-1}\right) & =g_{2}\left(a_{1}, \ldots, a_{n-1}\right)+\delta h\left(a_{1}, \ldots, a_{n-1}\right) \\
& =g^{\prime}\left(a_{1}, \ldots, a_{n-1}\right) .
\end{aligned}
$$

Now proceed recursively to build up an $\alpha$-coherent sequence $\left\{g_{r}^{\prime}\right\}$ with $g_{r}^{\prime} \in$ $C^{n-1}\left(T_{m_{r}, n_{r}}, T_{m_{r}, n_{r}}\right), g_{r}^{\prime}=g_{r+1}^{\prime}$ on $T_{m_{r}, n_{r}} \cap T_{m_{r+1}, n_{r+1}}$ and $\delta g_{r}^{\prime}=f_{r}$. It is true that the norms of the $g_{r}^{\prime}$ 's can "build up", but since $n_{r}<m_{r+1}$ after finitely many $r$, we can take $g_{r}^{\prime}=g_{r}$ after a finite number of $r$ 's. This completes the construction of the $g_{r}^{\prime}$.

Corollary 5.8. If $n_{r}<m_{r+1}$ for all but finitely many $r$, then $H^{n}\left(T^{\infty}, J_{\alpha}\right)=0$ for $n \geq 2$.

Proof. Use Theorem 5.7, Lemma 5.4 and Proposition 5.3.

The next result gives a partial converse to Corollary 5.8.

Theorem 5.9. Suppose that for all $r, m_{r}<m_{r+1} \leq n_{r}<n_{r+1}$, and that

$$
\sup _{r \geq 1}\left(n_{r}-m_{r}\right)=M<\infty \text {. }
$$

Then $H^{2}\left(T^{\infty}, J_{\alpha}\right) \neq 0$.

Proof. Trivially, $M_{\alpha}<\infty$, so that $\alpha$ is bounded. By Lemma 5.4, we just have to show that $H^{1}\left(T^{\infty}, R_{\alpha}\right) \neq 0$. Define $D_{r}: T_{m_{r}, n_{r}} \rightarrow T_{m_{r}, n_{r}}$ by:

$$
D_{r}\left(e_{i j}\right)=(j-i) e_{i j} .
$$

Then for $a \in T_{m_{r}, n_{r}}, D_{r}(a)=a b_{r}-b_{r} a$, where $b_{r}$ is the diagonal matrix:

$$
b_{r}=\left[\begin{array}{cccc}
1 & & & \\
& 2 & & \\
& & \ddots & \\
& & & n_{r}-m_{r}+1
\end{array}\right] \text {. }
$$

Thus $D_{r}$ is an inner derivation and

$$
\left\|D_{r}\right\| \leq 2\left\|b_{r}\right\| \leq 2\left(n_{r}-m_{r}+1\right) \leq 2(M+1) .
$$

Since $b_{r}$ is diagonal, each $D_{r}$ is multimodular. From the definition, $D_{r}$ and $D_{r+1}$ coincide on $T_{m_{r}, n_{r}} \cap T_{m_{r+1}, n_{r+1}}$. So $\left\{D_{r}\right\}$ is an $\alpha$-coherent sequence of derivations. By Proposition 5.6, there exists a multimodular derivation $D: T^{\infty} \rightarrow R_{\alpha}$ such that

$$
e_{r} D(a) e_{r}=D_{r}\left(e_{r} a e_{r}\right)
$$

for all $r$. 
Now suppose that $H^{1}\left(T^{\infty}, R_{\alpha}\right)=0$. Then $D$ is inner, and there exists $z \in R_{\alpha}$ such that for all $a \in T^{\infty}$ (with $\cdot$ the module action of $T^{\infty}$ on $R_{\alpha}$ ),

$$
D(a)=a \cdot z-z \cdot a .
$$

Since $D$ is multimodular, $D(b)=b z-z b=0$ for all $b \in B$ (noting that the . module action of $B$ on $R_{\alpha}$ is just normal multiplication). It follows that $z \in B$. Let $z_{r}=e_{r} z$. From (56), $D_{r}(a)=a z_{r}-z_{r} a$. Since (by the above) we also have $D_{r}(a)=a b_{r}-b_{r} a$, it follows that $z_{r}=b_{r}+\lambda_{r} e_{r}$ for some $\lambda_{r} \in \mathbb{C}$. By subtracting $\lambda_{1} I$ from $z$, we can suppose that $z_{1}=b_{1}$. The $\left(m_{2}, m_{2}\right)$ entry of $z$ is then $m_{2}-m_{1}+1$. But $z_{2}-b_{2}=\lambda_{2} e_{r}$. So $m_{2}-m_{1}+1=1+\lambda_{2}$, giving $\lambda_{2}=m_{2}-m_{1}$ and $\left(z_{2}\right)_{i}=\left(i-m_{2}+1\right)+\left(m_{2}-m_{1}\right)=i-m_{1}+1$. Thus the $\left(m_{3}, m_{3}\right)$ entry of $z_{3}$ is $m_{3}-m_{1}+1$, giving $\lambda_{3}=m_{3}-m_{1}$ and

$$
\left(z_{3}\right)_{i}=\left(i-m_{3}+1\right)+\left(m_{3}-m_{1}\right)=i-m_{1}-1 .
$$

By induction, $\left(z_{p}\right)_{i}=i-m-1$, and so, for all $p$,

$$
\|z\| \geq\left\|z_{p}\right\|=n_{p}-m_{1}+1 \rightarrow \infty
$$

as $p \rightarrow \infty$. This is a contradiction.

Note. A result of Erdos and Power ([7], p. 233) determines $H^{1}(A, X)$ when $A$ is a nest algebra $\subseteq B(H)$ and $X$ is a weakly closed $A$-submodule of $B(H)$. Another proof of their result (using the multimodular property) can be given along the following lines, where we give it in the case of $H^{1}\left(T^{\infty}, J_{\alpha}\right)$. No boundedness requirement on $\alpha$ is needed.

Firstly, $H^{1}\left(T^{\infty}, J_{\alpha}\right)=Z_{m}^{1}\left(T^{\infty}, J_{\alpha}\right) / \delta\left(C_{m}^{0}\left(T^{\infty}, J_{\alpha}\right)\right)$, where the subscript $m$ stands for multimodular. Using the Lance-Nielsen result and part of the preceding proof, we obtain

$$
Z_{m}^{1}\left(T^{\infty}, J_{\alpha}\right)=\left\{b \in B: a b-b a \in J_{\alpha} \text { for all } a \in T^{\infty}\right\} / \mathbb{C} I
$$

while

$$
\delta\left(C_{m}^{0}\left(T^{\infty}, J_{\alpha}\right)\right)=\left\{\mathbb{C} I+b: \quad b \in B \cap J_{\alpha}\right\} / \mathbb{C} I,
$$

Now $a b-b a \in J_{\alpha} \Leftrightarrow(a b-b a)_{i j}=0$ for all $a \in T^{\infty}$, where $m_{r} \leq i \leq j \leq n_{r}$ for some $r \Leftrightarrow e_{r} b=\lambda_{r} e_{r}$ for some $\lambda_{r} \in \mathbb{C}$ and all $r$. Let $Y=\bigcup\left\{\left[m_{r}, n_{r}\right]: r \geq 1\right\}$ (using integer intervals). Then $Y$ is the disjoint union of maximal integer intervals. Let $\mathcal{B}$ be the family of these intervals. Then

$$
Z_{m}^{1}\left(T^{\infty}, J_{\alpha}\right)=\{b \in B: \quad b \text { is constant on the members of } \mathcal{B}\} / \mathbb{C} 1 .
$$

Suppose $T^{\infty} \neq J_{\alpha}$. Then $\delta\left(C_{m}^{0}\left(T^{\infty}, J_{\alpha}\right)\right)$ can be identified with $\left\{b \in Z_{m}^{1}\left(T^{\infty}, J_{\alpha}\right)\right.$ : $b$ vanishes on the members of $\mathcal{B}\}$, and we obtain $H^{1}\left(T^{\infty}, J_{\alpha}\right)=\ell^{\infty}(\mathcal{B}) / \mathbb{C} 1$.

\section{REFERENCES}

1. Christensen, E., Derivations of nest algebras, Math. Ann. 229 (1977), 155-161. MR 56:6420

2. Connes, A., Classification of injective factors, Ann. of Math. 104 (1976), 73-115. MR 56:12908

3. Connes, A., On the cohomology of operator algebras, J. Functional Analysis 28 (1978), 248253. MR 58:12407

4. Davidson, K.R., Nest Algebras, Pitman Research Notes in Mathematics, Vol. 191, Longman Scientific and Technical, Harlow, England, 1988. MR 90f: 47062

5. Effros, E.G. and Kishimoto, A., Module maps and Hochschild-Johnson Cohomology, Indiana Math. J. 36 (1987), 257-276. MR 89b:46068 
6. Eilenberg, S. and Moore, J.C., Foundations of relative homological algebra, Mem. Amer. Math. Soc., No. 55 (1965). MR 31:2294

7. Erdos, J.A. and Power, S.C., Weakly closed ideals of nest algebras, J. Operator Theory 7 (1982), 291-235. MR 84a:47056

8. Gilfeather, F., Hopenwasser, A. and Larson, D.R., Reflexive algebras with finite width lattices: tensor products, cohomology, compact perturbations, J. Functional Analysis 55 (1984), 176199. MR 85g: 47062

9. Gilfeather, F. and Smith, R.R., Cohomology for operator algebras: cones and suspensions, Proc. London Math. Soc. 65 (1992), 175-198. MR 93i:46137

10. Gilfeather, F. and Smith, R.R., Cohomology for operator algebras: joins, Amer. J. Math. 116 (1994), 541-561. MR 95d:46077

11. Haagerup, U., All nuclear $C^{*}$-algebras are amenable, Invent. Math. 74 (1983), 305-319. MR 85g:46074

12. Helemskii, A.Ya., The homology of Banach and topological algebras, Kluwer, Dordrecht, 1989. MR 92d: 46178

13. Johnson, B.E., Cohomology in Banach algebras, Mem. Amer. Math. Soc., No. 127 (1972). MR 51:11130

14. Ringrose, J.R., Cohomology of operator algebras, Lecture Notes in Mathematics, vol. 247, Springer-Verlag, New York, 1972, pp. 355-433. MR 52:3983

15. Lance, E.C., Cohomology and perturbations of nest algebras, Proc. London Math. Soc. 43 (1981), 334-356. MR 83b:47053

16. Nielsen, J.P., Cohomology of some non-self-adjoint operator algebras, Math. Scand. 47 (1980), 150-156. MR 82k:47062

17. Paterson, A.L.T., Virtual diagonals and $n$-amenability for Banach algebras, Pacific J. Math. (to appear).

18. Sinclair, A.M. and Smith, R.R., Hochschild cohomology of von Neumann algebras, London Mathematical Society Lecture Notes Series 203, Cambridge University Press, Cambridge, 1995. MR 96d:46094

Department of Mathematics, University of Mississippi, University, Mississippi 38677

E-mail address: mmap@sunset.backbone.olemiss.edu

Department of Mathematics, Texas A\&M University, College Station, Texas 77843

E-mail address: rsmith@math.tamu.edu 